\title{
An $M$-Theory Perspective on Heterotic K3 Orbifold Compactifications
}

\author{
Michael Faux ${ }^{1}$, Dieter Lüst ${ }^{2}$ and Burt A. Ovrut ${ }^{3}$ \\ ${ }^{1}$ Departments of Mathematics and Physics \\ Columbia University \\ 2990 Broadway, New York, NY 10027 \\ ${ }^{2}$ Institut für Physik, Humboldt Universität \\ Invalidenstraße 110, 10115 Berlin, Germany \\ ${ }^{3}$ Department of Physics, University of Pennsylvania \\ Philadelphia, PA 19104-6396, USA
}

\begin{abstract}
We analyze the structure of heterotic $M$-theory on $K 3$ orbifolds by presenting a comprehensive sequence of $M$-theoretic models constructed on the basis of local anomaly cancellation. This is facilitated by extending the technology developed in our previous papers to allow one to determine "twisted" sector states in non-prime orbifolds. These methods should naturally generalize to four-dimensional models, which are of potential phenomenological interest.
\end{abstract}

PACS: 04.65.+e; 12.60.J; 11.25.-w; 11.10.Kk

Keywords: M-theory; Orbifolds; Strings; Anomalies 


\section{Introduction}

Orbifolds describe an interesting category of compactification schemes likely to provide useful physical models based on $M$-theory. The fundamental example of an $M$-theory orbifold, based on $S^{1} / \mathbf{Z}_{2}$, was presented in [1, 2], and examples based on $T^{4} / \mathbf{Z}_{2}$ were presented in [3, 4]. Since these incorporate nonperturbative aspects of related models, an algorithm for sifting through the various possibilities would complement and extend the perturbative string orbifold models classified more than ten years ago [5, 6]. Indeed, nonperturbative $M$-theory orbifolds could overcome the phenomenological shortcomings endemic in the string perturbative case.

In two previous papers [7, 8], we outlined much of the technology needed to ascertain microscopic properties of $M$-theory orbifolds, emphasizing the role of local anomaly cancellation in the determination of local "twisted" sector gauge groups and matter content. In those papers, as in this present one, we concentrate on the case of $S^{1} / \mathbf{Z}_{2} \times K 3$ orbifolds cases. In [7], we described special constraints which arise at fixed-plane intersections, and delineated the various contributions to both the quantum and inflow anomalies. In particular, we introduced the necessity for an intersection anomaly in orbifolds. This allows one to determine twisted gauge groups localized on odd-dimensional orbifold planes, which do not themselves support a local anomaly. Subsequent papers [10, 11] then supplied additional and complementary input to this technology. In [8], we also explained how fivebranes may mediate transitions linking different "phases" describing separate solutions to the local anomaly cancellation requirements.

In this paper, we extend our previous results and technology to include non-prime orbifolds. We present a detailed local analysis of anomaly cancellation in all four $S^{1} / \mathbf{Z}_{2} \times$ $K 3$ orbifolds describing degenerations of the sort $K 3 \rightarrow T^{4} / \mathbf{Z}_{M}$. (These exist for the cases $M=2,3,4$ and 6.) This rounds out the presentation in [7, 8] and complements the analysis in [11]. Some of the results in this paper were previously presented in [11]. However, our technology differs, and we also have new results which more fully unify the overall discussion. Specifically, we find a systematics associated with the orbifold projection of seven-dimensional gauge groups onto Cartan subgroups. This enables a discussion of possible phase transitions, and indicates smooth interconnections linking various possible phases of the moduli space.

We expect that intersection anomalies will play a crucial role in eventual studies of $M$-theory phenomenology. In this paper, we present a comprehensive list of models de-

\footnotetext{
${ }^{1}$ For a review on heterotic $K 3$ compactifications see e.g. [9].
} 
scribing portions of the low-energy moduli space corresponding to $M$-theory compactified on $S^{1} / \mathbf{Z}_{2} \times K 3$ orbifolds, and we discuss a number of technical and conceptual issues associated with these. The technology is developed with the expressed intent of enabling a search for phenomenologically more interesting compactifications to four dimensions. Our aim is to partially delineate how nonperturbative effects, particularly those involving fivebrane-mediated phase transitions, can modify phenomenological predictions based on perturbative orbifolds, and also to facilitate the search for a more fundamental description of $M$-theory.

Significant progress along these lines has already been achieved in heterotic $M$-theory models [13]. These are based on the Horava-Witten $S^{1} / \mathbf{Z}_{2}$ orbifold compactified to four dimensions on suitable Calabi-Yau threefolds $X$. Clearly, new $M$-theory models can be obtained by replacing these Calabi-Yau manifolds, and indeed all of $S^{1} / \mathbf{Z}_{2} \times X$, by more general non-perturbative orbifolds. This paper is a step in that direction.

Each of the $S^{1} / \mathbf{Z}_{2} \times T^{4} / \mathbf{Z}_{M}$ orbifolds describes a brane world involving a pair of parallel ten-planes linked by some number of parallel seven-planes with six-plane intersections. The seven-planes correspond to the $A_{M-1}$ singularities of the $K 3 \rightarrow T^{4} / \mathbf{Z}_{M}$ orbifold degenerations]. For reasons motivated in [11], one expects that each $A_{M-1}$ seven-plane should support an $S U(M)$ gauge theory. In that paper, consistent models were presented with this feature for each of $M=2,3$ and 4 , but not for the case $M=6$. For the case $M=6$, the authors of [11] present one model (with gauge group $S U(9) \times S U(3) \times S U(2)$ ) which requires that the secondary $A_{1}$ seven-planes support $U(1)$ gauge groups instead of $S U(2)$. In that case, the $U(1)$ factor is invisible in the infrared limit, and all anomalies do cancel.

An observation pointed out in [11] is that whenever infrared-visible $U(1)$ factors appear there exist non-cancelled anomalies. They present only one example of this case, which is an $M=4$ model with gauge group $E_{6} \times S O(10) \times S U(4) \times S U(2) \times U(1)$, which has non-cancelled local gravitational, mixed and gauge anomalies in both the abelian factors as well as the nonabelian factor. In this paper, we present a new class of $M=6$ models, with gauge group $E_{6} \times S U(8) \times S U(2) \times U(1)^{2+b}$, for $0 \leq b \leq 5$, in which all gravitational and mixed anomalies, as well as all nonabelian gauge anomalies do cancel. Furthermore, our model has $S U(N)$ gauge groups on each of the $A_{N-1}$ seven-planes, including all secondary planes. We see the (highly nontrivial) cancellation of all gravitational, mixed and nonabelian gauge anomalies as an encouraging sign. We relegate the resolution of the remaining abelian gauge anomalies to a future paper.

This paper is structured as follows. In Section 2, we review the basics of $M$-theory orbifold anomaly analyses for the case of prime orbifolds. We also introduce streamlined

\footnotetext{
${ }^{2}$ The four dimensions transverse to the seven-planes corresond to directions along the $K 3$.
} 
conventions relative to our previous papers and present a generalized proof, based on anomaly cancellation, that certain orbifold fixed planes can sequentially absorb (or emit) fivebranes from (or into) the eleven-dimensional bulk while maintaining consistency. This requires that the fivebrane tensor fields involve interesting dynamics which allow a localized version of the Green-Schwarz mechanism. In Section 3, we extend our technology to include nonprime orbifolds. In Section 4, we explain how to reduce the anomaly calculation to a set of very efficient equations which only involve rational data parameterizing the orbifold geometry, magnetic and electric charges, and various group theory factors. This enables an algorithmic search for consistent orbifold vertices. In Section 5, we explain the various consistent models which we have obtained by applying our technology to the orbifolds mentioned above, and explain how these models are interconnected by virtue of fivebrane-mediated phase transitions. In Section 6, we explain the microscopic details of the models described in section 5. This is done by use of "vertex diagrams" and "ladder diagrams" which conveniently depict the salient geometric properties. We also include an appendix which tabulates all of the rational parameters needed to verify the consistency of each of the vertices presented in the main text.

\section{Prime Orbifolds}

In this paper, we concentrate on the four $S^{1} / \mathbf{Z}_{2} \times T^{4} / \mathbf{Z}_{M}$ orbifold limits of $S^{1} / \mathbf{Z}_{\mathbf{2}} \times K 3$, corresponding to $M=2,3,4$ and 6 . We refer to these succinctly as the $\mathbf{Z}_{2}, \mathbf{Z}_{3}, \mathbf{Z}_{4}$ and $\mathbf{Z}_{6}$ orbifolds, respectively. The first two of these are prime orbifolds (since $M$ is a prime integer in these cases) and the second two are non-prime. Each of these orbifolds has a pair of fixed ten-planes (associated with the universal $\mathbf{Z}_{2}$ factor) and, additionally, some number of parallel fixed seven-planes (associated with the factor $\mathbf{Z}_{M}$ ). Each of the seven-planes transverally intersects each of the ten-planes once, at a particular six-plane.

For prime orbifolds, the fixed seven-planes are indistinguishable. For instance, the $\mathbf{Z}_{2}$ orbifold has sixteen and the $\mathbf{Z}_{3}$ orbifold has nine of these. For non-prime orbifolds, there are distinguishable sets of fixed seven-planes. (We describe this in more detail in the following section.) As a consequence, the analysis of local anomalies in non-prime orbifolds is more complicated, and requires a greater systematics, than in the case of prime orbifolds. In this section, we recapitulate the systematics involved in analyzing

prime orbifolds, so that we can readily extend the analysis to include both prime and non-prime orbifolds in subsequent sections.

Local anomaly matching on the two ten-planes uniquely determines that these planes each support a ten-dimensional $E_{8}$ super Yang-Mills multiplet [2]. As explained in [8], 
additional, more stringent, constraints follow from local anomaly matching at each of the six-planes describing intersections of the ten-planes and the seven-planes. Resolving these constraints allows one to determining various elements of data. These include local breaking patterns for the $E_{8}$ factors, a magnetic charge (denoted $g$ ) associated with each six-plane, electric parameters (denoted $\eta$ and $\rho$ ) associated with seven-dimensional ChernSimons interactions [ [ , a seven-dimensional gauge group $\tilde{\mathcal{G}}_{7}$, and additional "twisted" fields. The twisted fields can include seven-dimensional Yang-Mills multiplets and also six-dimensional $N=1$ vector, hyper and tensor multiplets.

In general, on a given ten-plane, the associated $E_{8}$ factor is broken to a subgroup $E_{8} \rightarrow \mathcal{G} \subset E_{8}$ on the embedded intersection six-planes by the nontrivial action of $\mathbf{Z}_{M}$ on the components of the $E_{8}$ multiplet. We assume that $\mathcal{G}$ is a direct product of simple and/or $U(1)$ factors. We define $p$ as the number of such factors, so we can write $E_{8} \rightarrow \mathcal{G}_{1} \times \cdots \times \mathcal{G}_{p}$. The field strength associated with $\mathcal{G}_{i}$ is described by a matrix-valued two-form $F_{i}$. The twisted gauge group $\tilde{\mathcal{G}}_{7}$ can likewise be broken on the intersection six-planes to a subgroup (for instance, its Cartan subgroup) by the action of the universal $\mathbf{Z}_{2}$ factor. Thus, we can write $\tilde{\mathcal{G}}_{7} \rightarrow \tilde{\mathcal{G}}_{1} \times \cdots \times \tilde{\mathcal{G}}_{q}$, where we define $q$ as the number of such factors. The field strength associated with $\tilde{\mathcal{G}}_{j}$ is described by a matrix-valued two-form $\mathcal{F}_{j}$.

On a given intersection six-plane, the anomaly involves only those gauge field strengths associated with one of the $E_{8}$ factors and one of the $\tilde{\mathcal{G}}_{7}$ factors. We concentrate on a particular six-plane and safely suppress any labelling which distiguishes which six-plane we are considering. It is convenient to describe all of the $p+q$ field strengths collectively using a notation $F_{I}$, where $F_{I}=\left\{F_{1}, \ldots, F_{p}, \mathcal{F}_{1}, \ldots, \mathcal{F}_{q}\right\}$. In this case, the anomaly on a given six-plane is described by an eight-form polynomial which we can write as

$$
\widehat{I}=A \operatorname{tr} R^{4}+B\left(\operatorname{tr} R^{2}\right)^{2}+C^{I} \operatorname{tr} R^{2} \wedge \operatorname{tr} F_{I}^{2}+D^{I J} \operatorname{tr} F_{I}^{2} \wedge \operatorname{tr} F_{J}^{2}+E^{I} \operatorname{tr} F_{I}^{4}
$$

where the various coefficients $A, B, C^{I}, D^{I J}$ and $E^{I}$ are determined by summing each of the various quantum and inflow anomalies as explained in [8].

If there are no tensor fields in the local twisted spectrum, then the anomaly (2.1) must vanish identically. In this case, each of the coefficients $A, B, C^{I}, D^{I J}$ and $E^{I}$ must independently vanish. If there is one twisted tensor field, then a local Green-Schwarz mechanism can cancel the anomaly provided $\widehat{I}$ factorizes as a product of two identical four forms. In this case, the requirement that $\widehat{I}$ be a complete square follows from supersymmetry (since tensor multiplets contain anti-self dual tensor fields). In the general case, if there are $n_{T}$ local tensor multiplets, then anomaly matching requires that $\widehat{I}$ be a

\footnotetext{
${ }^{3}$ We refer the reader to [8] for a discussion of each of the parameters $g, \eta$ and $\rho$.
} 
sum of $n_{T}$ complete squares. In any case, cancellation or factorization of $\widehat{I}$ each requires that $A=0$ and $E^{I}=0$, since these correspond to non-factorizable terms in the anomaly.

Henceforth, we assume that $A=0$ and $E^{I}=0$, as required by the preceeding discussion. We analyze the requirement $A=0$ in more detail in the next section, and discuss the $E^{I}=0$ requirement during the course of this section. The remaining coefficients are given by

$$
\begin{aligned}
B & =\frac{1-\eta}{2 f}-\frac{n_{T}}{8} \\
C^{I} \operatorname{tr} F_{I}^{2} & =\left(\frac{g}{2}+\frac{\eta}{f}\right) \operatorname{tr} F^{2}+\frac{1}{6} \operatorname{trace}^{\prime} F^{2}+\frac{1}{6} \operatorname{trace} \mathcal{F}^{2}+\rho \operatorname{tr} \mathcal{F}_{7}^{2} \\
D^{I J} \operatorname{tr} F_{I}^{2} \wedge \operatorname{tr} F_{J}^{2} & =-\frac{g}{2}\left(\operatorname{tr} F^{2}\right)^{2}-\frac{2}{3} \operatorname{trace} F^{4}-2 \rho \operatorname{tr} F^{2} \wedge \operatorname{tr} \mathcal{F}_{7}^{2}-\frac{2}{3} \operatorname{trace} \mathcal{F}^{4} .
\end{aligned}
$$

Various mnemonics are involved in (2.2). Traces denoted "tr" describe group theoretic reduction of the $E_{8}$ trace into traces over the various subgroup factors $F_{i}$, defined above. Traces denoted "trace" are quantum traces, which include an extra minus sign when hypermultiplets are involved. Traces denoted "trace'" are weighted quantum traces which also involve divisors related to orbifold-plane multiplicity. For the prime orbifolds, this is defined simply

$$
\operatorname{trace}^{\prime} F^{r}=\frac{1}{f} \sum_{i=1}^{p} \sum_{\mathcal{R}}(-1)^{2 J} \operatorname{tr}_{\mathcal{R}} F_{i}^{r},
$$

where $f$ is the number of seven-planes for the orbifold in question, $r$ is any arbitrary power, and $J$ is the highest spin in the multiplet contributing the anomalous coupling. (Thus, for hypermultiplets $J=1 / 2$ and for vector multiplets $J=1$.) Terms in (2.2) involving $F$ describe anomalies arising from ten-dimensional fields. Terms involving $\mathcal{F}$ describe anomalies due to additional six or seven dimensional twisted fields f. Finally, terms involving $\mathcal{F}_{7}$ are inflow contributions involving only the seven-dimensional twisted group $\tilde{\mathcal{G}}_{7}$. Note that the cancellation of $E^{I}$ is implicit in the last equation of (2.2), since the right hand side can only factorize if this condition is satisfied.

If $n_{T}=0$, then we require that $B=C^{I}=D^{I J}=0$. If $n_{T}=1$ then, according to the discussion in the preceeding paragraph, we have a weaker requirement that the anomaly

\footnotetext{
${ }^{4}$ Note that we have scaled $\eta$ relative to the convention used in [8], such that $Y_{4}=\frac{1}{4 \pi}\left(-\frac{\eta}{2 f} \operatorname{tr} R^{2}+\right.$ $\rho \operatorname{tr} \mathcal{F}^{2}$ ). In this way, $\eta$ invariably turns out to be unity.

${ }^{5}$ Since the twisted fields generically transform under both $\mathcal{G}$ and $\tilde{\mathcal{G}}_{7}$, the terms in (2.2) mnemonically involving $\mathcal{F}$ will, in fact, include contributions from both $F_{i}$ and $\mathcal{F}_{i}$.
} 
factorize as a complete square. This is only possible if

$$
4 B D^{I J}=C^{I} C^{J}
$$

Since this requirement includes the stronger case where all coefficients vanish, equation (2.4) describes a generic constraint valid when $n_{T} \leq 1$.

Horizontal Hierarchy:

Note that if we locally increment the hypermultiplet multiplicity and the tensor multiplet multiplicity each by one gauge singlet, and simultaneously increment the magnetic charge by one such that $n_{H} \rightarrow n_{H}+1, n_{T} \rightarrow n_{T}+1$ and $g \rightarrow g+1$, then equation (2.2) tells us that

$$
\begin{aligned}
B & \longrightarrow B-\frac{1}{8} \\
C^{I} \operatorname{tr} F_{I}^{2} & \longrightarrow C^{I} \operatorname{tr} F_{I}^{2}+\frac{1}{2} \operatorname{tr} F^{2} \\
D^{I J} \operatorname{tr} F_{I}^{2} \wedge \operatorname{tr} F_{J}^{2} & \longrightarrow D^{I J} \operatorname{tr} F_{I}^{2} \wedge \operatorname{tr} F_{J}^{2}-\frac{1}{2}\left(\operatorname{tr} F^{2}\right)^{2}
\end{aligned}
$$

Furthermore, $A=0$ and $E^{I}=0$ are invariant under this shift as well. Therefore, if we substitute the modified values of the coefficients back into (2.1), we determine

$$
\begin{aligned}
\widehat{I} & \longrightarrow \widehat{I}-\frac{1}{8}\left(\operatorname{tr} R^{2}\right)^{2}+\frac{1}{2} \operatorname{tr} R^{2} \wedge \operatorname{tr} F^{2}-\frac{1}{2}\left(\operatorname{tr} F^{2}\right)^{2} \\
& =\widehat{I}-\frac{1}{8}\left(\operatorname{tr} R^{2}-2 \operatorname{tr} F^{2}\right)^{2} .
\end{aligned}
$$

So we observe that the net anomaly changes by the addition of one complete square. But, since the local twisted sector changes by the addition of one tensor multiplet, the net anomaly can be cancelled by a local Green-Schwarz mechanism mediated by the anti selfdual tensor field in this multiplet. We interpret this process as describing the absorption of a fivebrane onto the relevant intersection six-plane.

This analysis generalizes to include the absorption of any integer number $M$ of fivebranes. In this general case, we have $n_{H} \rightarrow n_{H}+M, n_{T} \rightarrow n_{T}+M$ and $g \rightarrow g+M$, and we determine

$$
\begin{aligned}
\widehat{I} & \longrightarrow \widehat{I}-\frac{M}{8}\left(\operatorname{tr} R^{2}-2 \operatorname{tr} F^{2}\right)^{2} \\
& =\widehat{I}-\sum_{i=1}^{M} \frac{1}{8}\left(\operatorname{tr} R^{2}-2 \operatorname{tr} F^{2}\right)^{2}
\end{aligned}
$$

So, regardless of how many fivebranes we sequentially pile onto the six-plane, we can maintain anomaly freedom at the vertex provided each successive tensor multiplet in- 


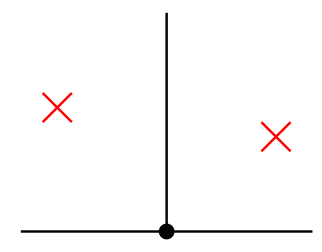

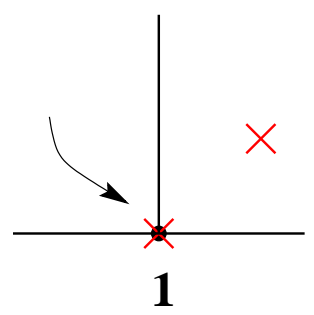

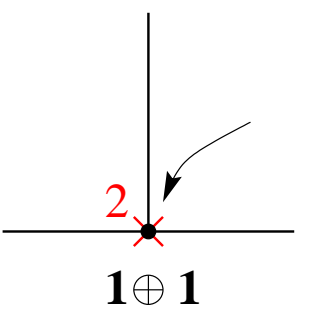

Figure 1: Any number of fivebranes can sequentially attach to a consistent intersection six-plane while retaining anomaly cancellation. Gauge-singlet twisted hypermultiplets are indicated by labels beneath the vertex.

dependently mediates a Green-Schwarz mechanism. The process of two fivebranes successively absorbed onto a particular intersection six-plane is depicted in Figure 1, which employs the sort of "vertex-diagrams" which were introduced and explained in [8].

We aim to classify sets of consistent local vertices. For reasons also explained in [8], these are conveniently assembled into arrays such that each column contains vertices with a specific number $n_{T}$ of twisted tensors, and each row contains vertices with a specific value of the difference $g-n_{T}$, where $g$ is the magnetic charge. The consistent sequential absorption of fivebranes therefore implies a horizontal motion in such an array of vertices, and we refer to the set of solutions in a given row of this array as describing a horizontal hierarchy.

\section{Non-Prime Orbifolds}

Non-prime orbifolds have more than one type of fixed plane. Hyperplanes fixed under the full discrete group we call primary. Secondary fixed planes are invariant under some subgroup of $\mathbf{Z}_{M}$ but comprise nontrivial multiplets under $\mathbf{Z}_{M}$. For instance, the $\mathbf{Z}_{4}$ orbifold has four primary fixed planes invariant under $\mathbf{Z}_{4}$ and six additional secondary planes, each describing a $\mathbf{Z}_{4}$ doublet of planes invariant under $\mathbf{Z}_{2} \subset \mathbf{Z}_{4}$. Finally, the $\mathbf{Z}_{6}$ orbifold has one primary fixed plane invariant under $\mathbf{Z}_{6}$, four $\mathbf{Z}_{3} \subset \mathbf{Z}_{6}$ secondary planes assembled as $\mathbf{Z}_{6}$ doublets and five more $\mathbf{Z}_{2} \subset \mathbf{Z}_{6}$ secondary planes assembled as $\mathbf{Z}_{6}$ triplets.

For the case of prime orbifolds, it is straightforward to determine the untwisted contribution to the local anomaly anomaly via index theorems and then distributing this result uniformly over the indis-

\footnotetext{
${ }^{6}$ By "untwisted" we refer to the local contribution due to nonlocalized fields, such as the bulk supergravity fields and (for the case of the six-dimensional anomalies) the ten-dimensional fields.
} 
tinguishable fixed planes. For the case of nonprime orbifolds, the untwisted contribution is less straightforward to determine due to the distinguishability of the fixed planes. As explained in [11, it is nevertheless clear how to resolve this problem. In a nonprime orbifold, the secondary planes are each identical to a primary fixed plane in a corresponding prime orbifold. (For instance, in the case of the $\mathbf{Z}_{6}$ orbifold the secondary $\mathbf{Z}_{3} \subset \mathbf{Z}_{6}$ fixedplanes are locally identical to the primary fixed planes in the $\mathbf{Z}_{3}$ orbifold.) But, since we know the untwisted anomaly for the prime orbifolds, we can readily determine the total anomaly due to all secondary fixed-planes in the nonprime orbifolds. To determine the untwisted contribution to the local anomaly on the primary fixed planes of a nonprime orbifold, we first determine the total anomaly via index theorems, and then subtract off the net contribution from all of the secondary fixed-planes. What remains is then uniformly distributed over the set of primary fixed-planes, which are indistinguishable.

It is straightforward to systemetize the process described in the previous paragraph. However, the analysis is facilitated if we first define a few useful conventions. We refer to a primary fixed-plane as a "1-type" fixed-plane and a secondary fixed-plane as "2type". If there are two distingishable types of secondary fixed-planes (as in the case of the $\mathbf{Z}_{6}$ orbifold), then one of these is denoted "2-type" and the other is denoted "3-type", with the distintion left as a choice of convention. And so forth. We then define $N_{n}$ as the number of $n$-type fixed-planes in the orbifold in question and $B_{n}$ as the number of primary-fixed planes for a prime orbifold of $n$-type. Finally, $h_{(n)}$ is the number of (untwisted) hypermultiplets derived from the bulk supergravity in a prime orbifold of $n$-type 7 . For each type of fixed plane, we can then define an "effective" number of $n$-type fixed planes as

$$
f_{(n)}=\frac{N_{1}}{N_{n}} B_{n}
$$

Each of the parameters defined so far plays a special role in the determination of the local anomalies. Also useful are the "effective" fixed plane multiplicity and the "net effective hypermultiplet ratio", respectively defined by

$$
\begin{aligned}
f & \equiv\left(\frac{1}{f_{(1)}}-\sum_{n \neq 1} \frac{1}{f_{(n)}}\right)^{-1} \\
\mathcal{H} & =\frac{h_{(1)}}{f_{(1)}}-\sum_{n \neq 1} \frac{h_{(n)}}{f_{(n)}} .
\end{aligned}
$$

Furthermore, the $\mathbf{Z}_{M}$ projection can act to leave different subgroups $\mathcal{G}^{(n)}$ invariant at the different $n$-type fixed planes, owing to the fact that on secondary planes only a subgroup

\footnotetext{
${ }^{7}$ Note that in addition to the untwisted hypermultiplets there is always one untwisted tensor multiplet asociated with the bulk supergravity.
} 
of $\mathbf{Z}_{M}$ induces the projection on the $E_{8}$ root lattice. The relevant projection is partially accounted for by the multiplicities of vector and hyper multiplets which remain from the $E_{8}$ fields on the fixed six-planes of $n$-type. These are defined as $V^{(n)}$ and $H^{(n)}$, respectively.

Cancellation of the $\operatorname{tr} R^{4}$ anomaly locally, on a given intersection six-plane, requires

$$
n_{H}-n_{V}=30 g-29 n_{T}+P+Q
$$

where $P$ is the supergravity contribution and $Q$ is the contribution deriving from the ten-dimensional $E_{8}$ fields. These are given by

$$
\begin{aligned}
P & =\frac{122}{f}-\frac{\mathcal{H}}{2} \\
Q & =\frac{1}{f_{(n)}}\left(V^{(1)}-H^{(1)}\right)-\sum_{n \neq 1} \frac{1}{f_{(n)}}\left(V^{(n)}-H^{(n)}\right) .
\end{aligned}
$$

Note that equation (3.3) is the requirement that $A=0$, as described in the previous section.

For each of the four $S^{1} / \mathbf{Z}_{2} \times T^{4} / \mathbf{Z}_{M}$ orbifolds, the parameters $N_{n}, B_{n}, h_{(n)}$ and $f_{(n)}$ for each type of fixed plane, and also the values of $f, \mathcal{H}$ and $P$ which can be derived from these using (3.2) and (3.4), are listed in Table 1. From the data exhibited in Table 1, we can roughly characterize the orbifold geometry in each case by the pictures shown in Figure 2.

In the general case, the anomaly is described by equation (2.2). However, in the case of nonprime orbifolds the weighted quantum trace should include the relevant secondaryplane subractions. As a result, we modify equation (2.3), replacing it with the following expression,

$$
\operatorname{trace}^{\prime} F^{r} \equiv \sum_{i=1}^{p} \sum_{\mathcal{R}}\left((-1)^{2 J} \frac{1}{f_{(1)}} \operatorname{tr}_{\mathcal{R}_{(1)}} F_{i}^{r}-\sum_{n \neq 1} \frac{1}{f_{(n)}}(-1)^{2 J} \operatorname{tr}_{\mathcal{R}_{(n)}} F_{i}^{r}\right)
$$

Note that for the case of prime orbifolds, $f_{(1)}=f$ is the only fixed-plane multiplicity parameter, so that in that case (3.5) is identical to (2.3). Thus, equation (3.5) incorporates the case of prime orbifolds.

The horizontal hierarchy is also valid in the case of non-prime orbifolds. This is because the shift (2.5) in the anomaly only involves the first terms in (2.2), which, in turn, only depend on local physics at the prime orbifold point (i.e. they do not involve the secondary subtractions). Thus, in the general case, available fivebranes can move to and consistently wrap any fixed six-plane in the orbifold. As a corollary, it is possible for six-planes to emit these fivebranes back into the bulk. Alternatively, it is possible that 
$\mathbf{Z}_{2}$ Orbifold:

\begin{tabular}{|cc|cc|cc|}
\hline$n$ & $P_{n}$ & $N_{n}$ & $B_{n}$ & $h_{(n)}$ & $f_{(n)}$ \\
\hline \hline 1 & $\mathbf{Z}_{2}$ & 16 & 16 & 4 & 16 \\
\hline \multicolumn{4}{c}{$f=16 \quad \mathcal{H}=\frac{1}{4} \quad P=\frac{15}{2}$} \\
\hline
\end{tabular}

$\mathbf{Z}_{3}$ Orbifold:

\begin{tabular}{|cc|cc|cc|}
\hline$n$ & $P_{n}$ & $N_{n}$ & $B_{n}$ & $h_{(n)}$ & $f_{(n)}$ \\
\hline \hline 1 & $\mathbf{Z}_{3}$ & 9 & 9 & 2 & 9 \\
\hline \multicolumn{4}{r}{$f=9 \quad \mathcal{H}=\frac{2}{9} \quad P=\frac{121}{9}$}
\end{tabular}

$\mathbf{Z}_{4}$ Orbifold:

\begin{tabular}{|cc|cc|cc|}
\hline$n$ & $P_{n}$ & $N_{n}$ & $B_{n}$ & $h_{(n)}$ & $f_{(n)}$ \\
\hline \hline 1 & $\mathbf{Z}_{4}$ & 4 & 4 & 2 & 4 \\
2 & $\mathbf{Z}_{2}$ & 6 & 16 & 4 & $\frac{32}{3}$ \\
\hline \multicolumn{3}{c}{$f=\frac{32}{5} \quad \mathcal{H}=\frac{1}{8} \quad P=19$}
\end{tabular}

$\mathbf{Z}_{6}$ Orbifold:

\begin{tabular}{|cc|cc|cc|}
\hline$n$ & $P_{n}$ & $N_{n}$ & $B_{n}$ & $h_{(n)}$ & $f_{(n)}$ \\
\hline \hline 1 & $\mathbf{Z}_{6}$ & 1 & 1 & 2 & 1 \\
2 & $\mathbf{Z}_{3}$ & 4 & 9 & 2 & $\frac{9}{4}$ \\
3 & $\mathbf{Z}_{2}$ & 5 & 16 & 4 & $\frac{16}{5}$ \\
\hline \multicolumn{4}{c}{$f=\frac{144}{35}$} & $\mathcal{H}=-\frac{5}{36}$ & $P=\frac{535}{18}$
\end{tabular}

Table 1: Some of the orbifold data associated with the four $S^{1} / \mathbf{Z}_{2} \times K 3$ orbifolds. 

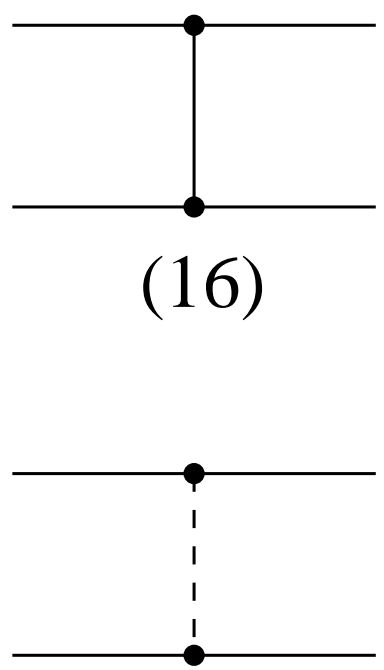

(9)
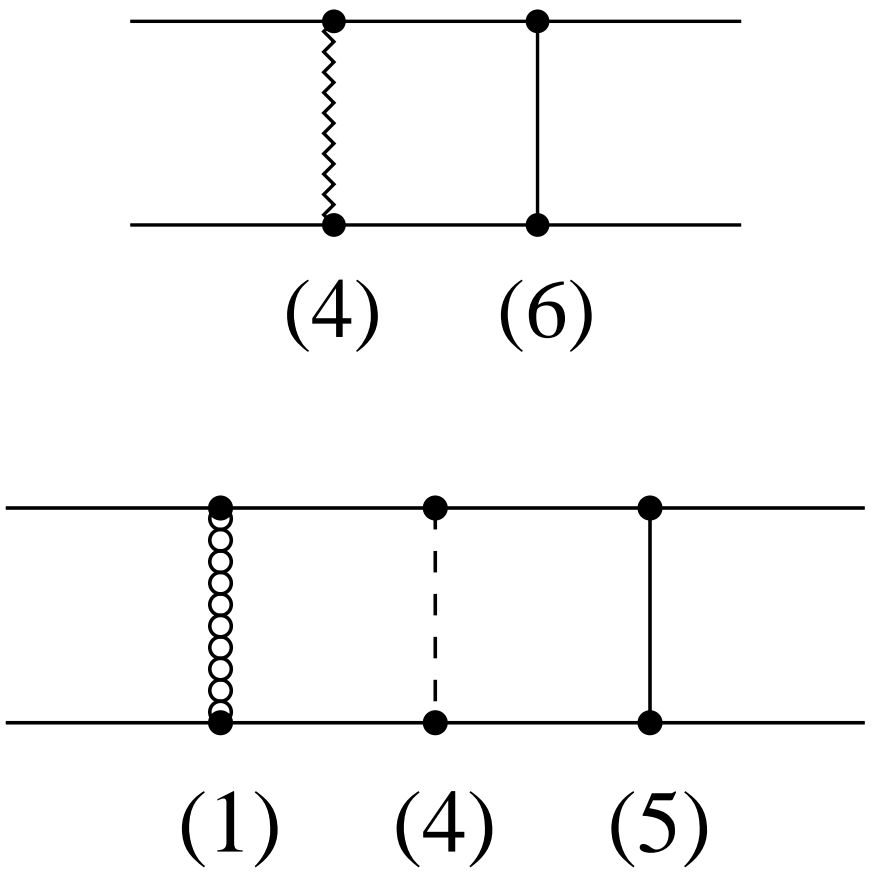

Figure 2: Orbifold fixed-plane geometry for the four $S^{1} / \mathbf{Z}_{2} \times T^{4} / \mathbf{Z}_{M}$ orbifolds. Top to bottom, we depict the cases $M=2,3,4$ and 6 . Horizontal lines are ten-planes, vertical lines are seven-planes, and the intersections are six-planes. Solid, dotted, jagged and bubbly lines respectively denote $\mathbf{Z}_{2}, \mathbf{Z}_{3}, \mathbf{Z}_{4}$ and $\mathbf{Z}_{6}$ fixed planes. Multiplicities are shown by the numbers in parentheses. 
a wrapped fivebrane (which is more properly identified as a torsion-free sheaf [14]) can smoothly connect on moduli space to a small gauge instanton. In this case, the $E_{8}$ factor is broken to a subgroup, the twisted tensor field is replaced with various hypermultiplet moduli, and the magnetic charge increases by one unit. Fractional transitions are also possible, in which the fivebrane data is distributed over some number of six-planes.

Thus, it is sensible to seek consistent orbifold vertices which are linked in moduli space to vertices without twisted tensor fields (i.e. $n_{T}=0$ ). In this case, all data is resolved by imposing that the anomaly vanish identically. The most basic of these solutions harbors no gauge instanton. In that case, the local magnetic charge assumes a minimal value which, for primary orbifold points, is given by $g_{\min }=-12 / f$. More interesting solutions have nontrivial breaking of the $E_{8}$ factor. In these cases $g>-12 / f$, and the difference $g-g_{\text {min }}$ describes a topological charge of the small instanton (or toron) residing on the fixed-plane.

\section{Local Anomaly Cancellation}

In order to determine the hierarchically-linked sector of moduli space defined in the preceeding paragraph, for each orbifold compactification under consideration, we should first determine anomaly-free vertices with $n_{T}=0$. Specifically, we seek sets of magnetic and electric charges, as well specific $E_{8}$ breaking patterns and twisted hypermultiplet representation content, which ensures that $A=0$ (i.e. equation (3.3)) and also ensures that each of the coefficients $B, C^{I}$ and $D^{I J}$ defined in equation (2.2) vanishes identically. To systemetize such a search, equation (2.2) can be more conveniently re-expressed soley in terms of various multiplicities which appear in the untwisted and twisted spectrum, and in terms of rational indices which characterize representations. In this way, the anomaly cancellation requirement reduces to a set of (overconstrained) algebraic relations amongst various rational and integer parameters. To determine these relations, we require a few more conventional definitions.

Each representation of each group factor appears with a multiplicity. We define $n_{1}(\mathcal{R})$ as the number of vector multiplets minus the number of hypermultiplets transforming in the representation $\mathcal{R}$ of the group $\mathcal{G}_{I}$ in the spectrum corresponding to the primary orbifold points. (The relevant group factor $\mathcal{G}_{I}$ is invariably clear from the context.) Similarly, there are representation multiplicities associated with each secondary orbifold point. These are defined in analogy to $n_{1}(\mathcal{R})$. Thus, $n_{n}(\mathcal{R})$ is the number of vector multiplets minus the number of hypermultiplets transforming in the representation $\mathcal{R}$ in the spectrum corresponding to $n$-type fixed planes. Finally, the parameter $\tilde{n}(\mathcal{R})$ describes the representation 
content of the twisted spectrum in analogy to the definitions made above. We also need to decompose the $E_{8}$ factors in terms of the gauge group $\mathcal{G} \subset E_{8}$ which remains unbroken on the primary fixed points, using the relevant branching rule [15]. This is needed to determine the inflow anomaly. We therefore define $n_{0}(\mathcal{R})$ as the (nonnegative) multiplicity parameter associated with this decomposition.

It proves useful to define an "effective multiplicity parameter" for each representation which appears in the untwisted spectrum,

$$
\mathrm{z}(\mathcal{R}) \equiv \frac{1}{f_{(1)}} n_{1}(\mathcal{R})-\sum_{n \neq 1} \frac{1}{f_{(n)}} n_{n}(\mathcal{R})
$$

This definition usefully accounts for both the secondary subtractions as well as the appropriate distribution factors. It is also useful to define a number of "anomaly weight factors"

$$
\begin{aligned}
X_{I} & =\frac{1}{30} \sum_{\mathcal{R}} n_{0}(\mathcal{R}) \mathcal{I}_{2}(\mathcal{R}) \\
Z_{I}^{(4)} & =\sum_{\mathcal{R}}(\mathrm{z}(\mathcal{R})+\tilde{n}(\mathcal{R})) \mathcal{I}_{4}(\mathcal{R}) \\
Z_{I}^{(2)} & =\sum_{\mathcal{R}}(\mathrm{z}(\mathcal{R})+\tilde{n}(\mathcal{R})) \mathcal{I}_{2}(\mathcal{R}) \\
Z_{I}^{(2,2)} & =\sum_{\mathcal{R}}(\mathrm{z}(\mathcal{R})+\tilde{n}(\mathcal{R})) \mathcal{I}_{2,2}(\mathcal{R}) \\
Z_{I J}^{(X)} & =\sum_{\mathcal{R}_{I} \times \mathcal{R}_{J}}\left(\mathrm{z}\left(\mathcal{R}_{I} \times \mathcal{R}_{J}\right)+\tilde{n}\left(\mathcal{R}_{I} \times \mathcal{R}_{J}\right)\right) \mathcal{I}_{2}\left(\mathcal{R}_{I}\right) \mathcal{I}_{2}\left(\mathcal{R}_{J}\right)
\end{aligned}
$$

where the rational numbers $\mathcal{I}_{2}(\mathcal{R}), \mathcal{I}_{2,2}(\mathcal{R})$ and $\mathcal{I}_{4}(\mathcal{R})$ are representation indices for the representation $\mathcal{R}$, which are defined in [8]. The weight factors (4.2) encorporate the twisted multiplicities $\tilde{n}(\mathcal{R})$ in a way which facilitates an algorithmic search for consistent vertices.

In terms of the weight factors given in (4.2), we can more efficiently describe the anomaly coefficients defined in (2.2), expressing them soley in terms of the multiplicities, 
representation indices, and orbifold plane multiplicities. We find 8

$$
\begin{aligned}
B & =\frac{1-\eta}{2 f}-\frac{n_{T}}{8} \\
C^{I} & =\left(\frac{g}{2}+\frac{\eta}{f}\right) X_{I}+\frac{1}{6} Z_{I}^{(2)}+\rho_{I} \\
D^{I I} & =-\frac{1}{2} g X_{I}^{2}-\frac{2}{3} Z_{I}^{(2,2)}-2 \rho_{I} X_{I} \\
D^{I J} & =-\frac{1}{2} g X_{I} X_{J}-2 Z_{I J}^{(X)}-\rho_{(I} X_{J)} \quad, I \neq J,
\end{aligned}
$$

where $\rho_{I}=\rho$ if $\mathcal{G}_{I}=\mathcal{G}_{7}$ and $\rho_{I}=0$ otherwise. Equation (4.3) describes the anomaly completely in terms of rational data.

Cancellation of all anomalies is necessary when $n_{T}=0$. In this case, the requirement $B=0$ implies $\eta=1$, as is readily seen from equation (4.3). We henceforth assume that $\eta=1$. In this case, the complete set of anomaly cancellation requirements are given by (3.3), which ensures cancellation of the $\operatorname{tr} R^{4}$ anomaly, and also the following

$$
\begin{aligned}
Z_{I}^{(4)} & =0 \\
Z_{I}^{(2)} & =-3\left(g+\frac{2}{f}\right) X_{I}-6 \rho_{I} \\
Z_{I}^{(2,2)} & =-\frac{3}{4} g X_{I}^{2}-3 \rho_{I} X_{I} \\
Z_{I J}^{(X)} & =-\frac{1}{4} g X_{I} X_{J}-X_{(I} \rho_{J} \quad, I \neq J,
\end{aligned}
$$

which correspond, respectively, to the cancellation of $\operatorname{tr} F_{I}^{4}, \operatorname{tr} R^{2} \wedge \operatorname{tr} F_{I}^{2},\left(\operatorname{tr} F_{I}^{2}\right)^{2}$, and $\operatorname{tr} F_{I}^{2} \wedge \operatorname{tr} F_{J}^{2}$ anomalies.

Equation (4.4) is of central importance in this paper. The primary objective in our analysis, which is summarized in the next section, is to discover and classify consistent local vertices by solving equation (4.4). This means identifying permitted breaking patterns of the ten-dimensional $E_{8}$ gauge group, any extra seven-dimensional twisted gauge groups, and any extra twisted matter. In addition, this means identifying the appropriate magnetic charge $g$ associated with the vertex in question, and also to identify the remaining electric parameter $\rho$ associated with the intersecting seven-plane.

\section{Consistent Models}

In this section, we analyze each of the four $S^{1} / \mathbf{Z}_{2} \times T^{4} / \mathbf{Z}_{M}$ orbifolds and establish a classification scheme for the solutions to the anomaly matching problem. In each case

\footnotetext{
${ }^{8}$ Symmetrization has weight one, so that $\rho_{(I} X_{J)}=\frac{1}{2}\left(\rho_{I} X_{J}+\rho_{J} X_{I}\right)$.
} 
we obtain a set of vertices by finding solutions to the constraints listed in Section 4 . The vertices are represented by specialized "vertex diagrams" which are exhibited in the following section. The technical aspects of the anomaly analysis are summarized in the tables in the appendix. The interested reader is encouraged to work through the appendix to verify the anomaly matching for each of the cited vertices. In each case, we use the elemental vertices as building blocks which we assemble into a variety of global "models", represented by ladder diagrams, which are also explained in [8]. There is an extra constraint imposed on the global construction. Namely, the net magnetic charge, obtained by summing the values of $g$ on each of the six-planes and total number of (unitcharged) fivebranes living in the bulk, must vanish.

We must also specify the manner in which the universal $\mathbf{Z}_{2}$ projection acts on the sevendimensional fields. As explained in [8], there are three possibilities. The seven-dimensional fields necessarily comprise an adjoint Yang-Mills supermultiplet, which decomposes in six dimensions as one vector multiplet and one hypermultiplet, each transforming as the adjoint of $\tilde{\mathcal{G}}_{7}$. The universal $\mathbf{Z}_{2}$ acts to project out some of this six-dimensional field content. The first possibility is that this projects out the hypermultiplet half, leaving a six-dimensional adjoint vector multiplet. The second possibility is the opposite of this, whereby the vector multiplet is projected out, leaving a hypermultiplet. This second case is permitted only if $\tilde{\mathcal{G}}_{7}$ is identified with one gauge factor in the unbroken subgroup $\mathcal{G} \subset E_{8}$ supplied from ten-dimensions. (In this way, a gauge connection is supplied from ten-dimensions, allowing us to project out the seven-dimensional vector and still retain six-dimensional gauge invariance.) On our vertex diagrams, we respectively denote the first two possibilities by a $V$ or an $H$ label on a downward arrow next to the vertical line which depicts the seven-plane. The third possibility is that the universal $\mathbf{Z}_{2}$ acts to break $\tilde{\mathcal{G}}_{7}$ to a subgroup $\mathcal{H} \subset \tilde{\mathcal{G}}_{7}$. On our vertex diagrams, this case is denoted by an $\mathcal{H}$ next to the downward arrow. For our purposes, we only consider the special case where $\mathcal{H}$ is the Cartan subgroup of $\tilde{\mathcal{G}}_{7}$. To illustrate our vertex-diagram conventions, we depict the three possibilities in Figure 3 for the choice $\tilde{\mathcal{G}}_{7}=S U(N)$.

For the cases where $\tilde{\mathcal{G}}_{7}$ is broken to its Cartan subgroup, we need to understand how the projection acts on the hypermultiplets. We specialize the discussion to those cases where $\tilde{\mathcal{G}}_{7}=S U(N)$, so that the Cartan subgroup is $U(1)^{N-1}$. Clearly, we have $N-1$ surviving vector multiplets in this case. But there remains a certain freedom in the way that $\mathbf{Z}_{2}$ can act on the hypermultiplet half of the seven-dimensional fields. Characteristically, we use anomaly cancellation to resolve this question. What we typically find is a need for retaining precisely $2(N-1)$ hypermultiplets out of the original $N^{2}-1$. This can be realized if $\mathbf{Z}_{2}$ acts as a modified Weyl reflection on the $S U(N)$ root lattice. Under 

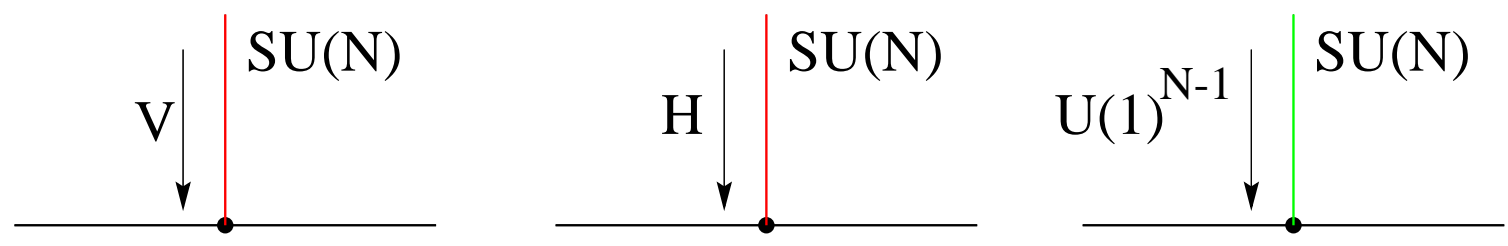

Figure 3: Three possibilities indicating different projections induced by the action of universal $\mathbf{Z}_{2}$ on $\tilde{\mathcal{G}}_{7}=S U(N)$. Respectively, the three diagrams indicate a projection onto an $S U(N)$ adjoint vector multiplet, an $S U(N)$ adjoint hypermultiplet, and the Cartan subgroup. In the latter case, the surviving vector and hypermultiplet content is explained in the text.

the modification, each Weyl-invariant root except for the one defining the Weyl reflection itself receives a parity flip. (Thus, we retain only one of the Weyl-invarant roots, while the remaining roots are halved.) Although this prescription is ad-hoc, it does illustrate how the needed accounting can be accomodated at the level of discrete projections. Obviously, a more fundamental description of $M$-theory is needed to provide a better explanation. But for the time being, anomaly matching is the best criteria which we have available.

Another issue concerns the electric parameter $\rho$. As it turns out, for any consistent primary $\mathbf{Z}_{M}$ orbifold vertex, this parameter is unity whenever the seven-dimensional gauge group is $S U(M)$, provided this group is unbroken by the universal $\mathbf{Z}_{2}$ projection. However, when this group is broken to its Cartan subgroup $U(1)^{M-1}$ we require $\rho=0$. Furthermore, in addition to the "conventional" vertices which involve $\tilde{\mathcal{G}}_{7}=S U(M)$ (either broken or unbroken by the universal $\mathbf{Z}_{2}$ ), there exist additional "unconventional" vertices, in which the seven-dimensional gauge group is $U(1)^{M-1}$ to begin with. In these cases, we also require $\rho=0$. As a rule of thumb, whenever a vertex supports an unbroken $S U(M)$ from the seven-plane then $\rho=1$. Otherwise $\rho=0$. As explained in [B], the possibility of two different values for $\rho$ poses an interesting riddle if we want to fully understand transitions which connect a " $\rho=0$ " phase to a " $\rho=1$ " phase. We have more to say about this riddle below.

We exhibit the microscopic details of the models in Section 6 and in the appendix. Here we offer a more superficial description of our results, and also explain how these relate to the results in [11]. A "model" is obtained by assembling a collection of consistent vertices in such a manner that the global constraints are also satisfied. There are two global constraints. The first is that the sum of all magnetic charges, including a unit positive charge for each bulk fivebrane, must vanish. This tells us that the sum of all of the independent orbifold vertex charges within a given orbifold must be nonpositive. In 
this way, the magnetic balance can be achieved, if necessary, by including fivebranes in the bulk. The second global constraint is that the electric parameters $\eta$ and $\rho$ associated with vertices linked by a particular seven-plane must be identical. This is because these parameters ostensibly derive from seven-dimensional interactions common to each of the two vertices in question. In this paper, we also impose a third global constraint: that there exists a sensible small-radius limit in which the untwisted states associated with individual vertices can be collectively resolved into a coherent low-energy untwisted spectrum. This implies that the untwisted spectrum associated to each six-plane intersection within a given ten-plane must combine into representations of a particular low-energy gauge group $\mathcal{G}_{\text {eff }} \subset E_{8}$ ?

The interesting models which satisfy our criteria are indicated in Table 2 . In that table we indicate, for each case, the effective gauge group obtained when all of the compact dimensions are shrunk to a point. In this way, we describe effective six-dimensional field theories with $N=1$ supersymmetry, with the indicated gauge groups, and with the indicated multiplicities $N_{H}, N_{V}$ and $N_{T}$, respectively describing the total nunber of hyper, vector and tensor multiplets, including both twisted and untwisted contributions. More complete descriptions of each of these models are given below. It may prove useful to examine the figures in Section 6 while reading this section. Some of these models, specifically Models 1,3 with $a=0,5$ and 9 with $b=0$ are not new to this paper, but were previously presented in [1]. This includes one model for each of the four K3 orbifolds and one special orbifold, namely Model 3, with the unconventional seven-dimensional $U(1)$ gauge group. The new models which we present in this paper fall into two categories. The first category includes "fully nonperturbative" models which include extra tensor multiplets and a completely unbroken $E_{8} \times E_{8}$ gauge group. For the cases of the $\mathbf{Z}_{2}, \mathbf{Z}_{3}$ and $\mathbf{Z}_{4}$ orbifolds, these new models are smoothly connected to the aforementioned models via phase transitions involving small instanton/fivebrane phase transitions. The second category of new models is given by Model 10, which is a new "perturbative" solution which permits us to discuss some interesting features of the $\mathbf{Z}_{6}$ orbifold.

Note that Model 2 and Model 4 with $a=16$ have the same gauge group, but have a different microscopic description. In Model 2, the seven-dimensional gauge group at each of the sixteen seven-planes is $S U(2)$, which is broken to $U(1)$ by the universal $\mathbf{Z}_{2}$ projection. (This is depicted in Figure 6.) In Model 4, on the other hand, the sevendimensional gauge groups are $U(1)$ to begin with. (This is shown in Figure 8.) As

\footnotetext{
${ }^{9}$ This precludes other potentially interesting situations which might describe orbifolds without sufficient moduli for the compact dimensions to be "shrunk" to a point, thereby describing universes with interesting "large" extra dimensions.
} 


\begin{tabular}{|c||c|c|ccc|}
\hline Orbifold & Model & Gauge Group & $N_{H}$ & $N_{V}$ & $N_{T}$ \\
\hline $\mathbf{Z}_{2}$ & 1 & $E_{7} \times S O(16) \times S U(2)$ & 500 & 256 & 1 \\
& 2 & $E_{8} \times E_{8} \times U(1)^{16}$ & 60 & 512 & 25 \\
& 3 & $E_{8} \times E_{7} \times S U(2) \times U(1)^{a}$ & $628+16 \mathrm{a}$ & $384+16 \mathrm{a}$ & 1 \\
& 4 & $E_{8} \times E_{8} \times U(1)^{a}$ & $44+16 \mathrm{a}$ & $496+16 \mathrm{a}$ & 25 \\
\hline $\mathbf{Z}_{3}$ & 5 & $E_{6} \times S U(9) \times S U(3)$ & 410 & 166 & 1 \\
& 6 & $E_{8} \times E_{8} \times U(1)^{16}$ & 62 & 514 & 25 \\
\hline $\mathbf{Z}_{4}$ & 7 & $S O(10) \times S U(8) \times S U(4) \times S U(2)$ & 370 & 126 & 1 \\
& 8 & $E_{8} \times E_{8} \times U(1)^{16}$ & 62 & 514 & 25 \\
\hline $\mathbf{Z}_{6}$ & 9 & $S U(9) \times S U(6) \times S U(3) \times S U(2) \times U(1)^{b}$ & $359+16 \mathrm{~b}$ & $115+16 \mathrm{~b}$ & 1 \\
& 10 & $E_{6} \times S U(8) \times S U(2) \times U(1)^{2+b}$ & $379+16 \mathrm{~b}$ & $135+16 \mathrm{~b}$ & 1 \\
& 11 & $E_{8} \times E_{8} \times U(1)^{16}$ & 62 & 514 & 25 \\
\hline
\end{tabular}

Table 2: A tabulation of the orbifold models discussed in the text. The unspecified parameters $a$ and $b$ can take any integer value such that $0 \leq a \leq 16$ and $0 \leq b \leq 5$. 
explained at length in [8], we envision a phase transition mediated by fivebranes which links Model 1 with Model 2, and another phase transition mediated by fivebranes which links Model 3 with Model 4. Model 1 and Model 2 then live on what we would call the "conventional" branch of moduli space, and Models 3 and 4 live on an "unconventional" branch. Models 3 and 4 are included in our survey because they correspond to the $\mathbf{Z}_{2}$ secondary plane used in Model 9.

For the $\mathbf{Z}_{3}$ and the $\mathbf{Z}_{4}$ orbifolds, we exhibit only the conventional solutions. In each case, there is a "perturbative" solution (with only one tensor multiplet) and a "nonperturbative" solution (with 25 tensor multiplets). In each case, the perturbative and the nonperturbative solutions are arguably smoothly connected on moduli space due to fivebrane-mediated phase transitions.

As we mentioned above, each of the "perturbative" models except for Model 10 were described in [11]. For the cases of the $\mathbf{Z}_{2}, \mathbf{Z}_{3}$ and $\mathbf{Z}_{4}$ orbifolds, these have fully conventional descriptions in the sense that each fixed seven-plane, both primary and secondary, supports a conventional $S U(N)$ gauge group which is unbroken by the universal $\mathbf{Z}_{2}$ projection. This is not so for the case of the $\mathbf{Z}_{6}$ orbifold, however. For instance, Model 9 requires that the $\mathbf{Z}_{2}$ secondary plane be unconventional. Specifically, in the microscopic description (shown in Figure 17), we see that each of the five $\mathbf{Z}_{2}$ secondary planes is similar to the $\mathbf{Z}_{2}$ primary planes in Model 3. This has the unconventional seven-dimensional $U(1)$ gauge group rather than the conventional $S U(2)$.

There is a model similar to Model 9, which we could call Model 9', with the same gauge group as Model 9 but with a different microscopic description. In Model $9^{\prime}$, the $\mathbf{Z}_{2}$ secondary planes do support the conventional $S U(2)$ gauge group on the $\mathbf{Z}_{2}$ secondary seven-planes, but these $S U(2)$ factors are projected to the $U(1)$ Cartan subgroup on each of two six-plane intersections. Model $9^{\prime}$ is more directly analogous to Model 10. Model 9, however, more accurately describes the situation presented in [11], since that paper does not address the possibility of unconventional projections. The difference between Model 9 and Model $9^{\prime}$ indicates a generic phenomenon: anomaly cancellation may indicate a need for either an unconventional seven-dimensional gauge group or a conventional gauge group with an unconventional projection. But in some cases, the local anomaly matching alone may not be sufficient to remove the ambiguity.

Model 10 is interesting because it provides a second "perturbative" $\mathbf{Z}_{6}$ solution. Notably, this case also requires either unconventional vertices or conventional vertices with unconventional projections. But in Model 10 it is the primary $\mathbf{Z}_{6}$ vertices rather than the secondary $\mathbf{Z}_{2}$ vertices which are distinguished: either the primary seven-plane supports the unconventional $U(1)^{5}$ gauge group or it supports the conventional $S U(6)$ gauge group 
which is projected to the $U(1)^{5}$ Cartan subgroup at the six-plane intersections. In the microscopic description of Model 10 (shown in Figure 18) we exhibit the first of these possibilities. (The second possibility could be called Model 10', and would be more directly analogous to Model 9.) Models $9^{\prime}$ and 10 are smoothly connected on moduli space to Model 11 via phase transitions involving small instanton/fivebrane transitions similar to those discussed in [8].

\section{Microscopic Details}

In this section, we present a more detailed analysis of the models described above. We do this by exhibiting a sequence of figures and tables which conveniently display the microscopic structure of each model. Specifically, for each of the four $K 3$ orbifolds we list a set of consistent vertices followed by a set of ladder diagrams which pictorially illustrate the microscopic structure of each of the models described in the previous section. We first give a description of how to interpret the figures associated with the $\mathbf{Z}_{2}$ orbifold. This makes the remaining figures self-explanatory.

A set of consistent vertices for the $\mathbf{Z}_{2}$ orbifold are shown in Figure 4a. The lefthand column in that figure depicts the "basic" vertices, without local tensor fields (i.e. $\left.n_{T}=0\right)$. The vertices in the right hand column have $n_{T}=1$, and are obtained from those in the left by the addition of a fivebrane. (Additional columns are generated by adding additional fivebranes.) The upper left hand vertex is the most basic of all, since this represents a situation which includes neither fivebranes nor small gauge instantons. As explained above, such a vertex has the smallest possible magnetic charge for this orbifold. Proceeding down the left-hand column are solutions in which the ten-dimensional $E_{8}$ gauge group is broken to subgroups, indicating the presence of small gauge instantons. In these cases, the magnetic charge is necessarily greater than the minimum value of $g_{\min }=-12 / f=-3 / 4$, and the difference $g-g_{\min }=g+3 / 4$ describes a topological charge of the instanton. In [8] we postulated that vertices degenerate in magnetic charge may be linked by phase transitions. Thus, the upper right-hand vertex in Figure 4a could mutate into the lower left-hand vertex (since these each have magnetic charge $g=1 / 4$ ) and vice-versa. Each of the vertices in Figure 4a are "conventional" because they have seven-dimensional gauge group $S U(2)$.

For the case of vertices with unbroken $E_{8}$ it is, in fact, impossible to decide on the basis of anomaly cancellation alone whether the seven-dimensional gauge group is actually $S U(2)$ broken to $U(1)$ by the projection, or whether the seven-dimensional gauge group is $U(1)$ itself. Nevertheless, the "conventional" choice of $S U(2)$ is suggested by the other 
vertices in the second and third row of Figure 4a, which are required by anomaly cancellation to support $S U(2)$ on the seven-planes. A commonality in this respect is seemingly important if the afformentioned phase transitions are to be possible.

In Figure 4b, we list additional "unconventional" vertices for the $\mathbf{Z}_{2}$ orbifold, which have seven-dimensional $U(1)$ gauge group rather than $S U(2)$. Once again, the left hand column includes "basic" vertices, which have $n_{T}=0$. The vertices in the right hand column each have $n_{T}=1$, and are obtained from those in the left by the addition of a fivebrane. Notice that there are two vertices in Figure $4 \mathrm{~b}$ with the minimal charge $-3 / 4$ and unbroken $E_{8}$ : one with a "vector" projection and the other with a "hyper" projection. This degeneracy reflects a limitation in the anomaly cancellation criteria. This is because the fermions in the $U(1)$ multiplet are gauge singlets. As a result, the only constraint available to us comes from gravitational anomalies, summarized by the requirement $A=0$. For the case with $n_{T}=0$ and unbroken $E_{8}$, this requires $n_{H}-n_{V}=\frac{1}{2}$, where $n_{H}$ and $n_{V}$ are the effective multiplicities of twisted hyper and vector multiplets. As explained in [7, 8], these include a factor of one-half for seven-dimensional fields. Thus, there are three possibilities: the conventional one and the two unconventional choices with opposite projections. For the case with hyper projection we have $n_{H}-n_{V}=1 / 2-0=1 / 2$. For the case with vector projection we need one additional (six-dimensional) twisted singlet hypermultiplet, so that $n_{H}-n_{V}=1-1 / 2=1 / 2$. The single hypermultiplet is indicatd by the 1 on the upper left-hand vertex in Figure $4 \mathrm{~b}$.

In Figures 5 and 6 we assemble the conventional $\mathbf{Z}_{2}$ vertices shown in Figure $4 a$ into global models along the lines described above. In Figures 7 and 8 we similarly assemble the unconventional $\mathbf{Z}_{2}$ vertices shown in Figure $4 \mathrm{~b}$ into global models. In each case, the two ten-planes are represented by parallel horizontal lines and the sixteen sevenplanes are collectively depicted by one vertical line. (Since the sixteen seven-planes are indistinguishable, we only depict one of these in the diagrams. A complete global picture of the relevant model is therefore obtained by visualizing sixteen copies of the associated figure.) Below each figure is a table in which all of the low energy hypermultiplets are listed in terms of their representation content.

There are alternatives to Models 3 and 4 obtained by making a change in the choice of $U(1)$ projection, replacing the $\mathrm{V}$ projection with an $H$ projection. This is due to the degeneracy, described above, which is indicated in Figure 4b. By changing one $V$ projection to an $H$ projection, the effect is to subtract one $U(1)$ factor from the effective gauge group, and to remove one vector and one hypermultiplet from the spectrum. The versions which we have illustrated in Models 3 and 4 have the maximal number of $U(1)$ factors. By altering the projections on some subset of the fixed-seven planes, we can 
construct any alternative to Models 3 and 4 with abelian factor $U(1)^{a}$ where $0 \leq a \leq 16$. Similar comments apply to Models 9 and 10.

On the basis of this discussion, the figures associated with the $\mathbf{Z}_{3}, \mathbf{Z}_{4}$ and $\mathbf{Z}_{6}$ orbifolds are sufficiently similar that we do not need to present separate detailed description of each case; the figures speak for themselves. The consistency of the vertices presented in each case can be verified by working through the numbers found in the tables in the appendix. Table 3 lists all of the figures which follow in this section. 


\begin{tabular}{|c|c|c|}
\hline Orbifold & Figure & Description \\
\hline \multirow[t]{6}{*}{$\mathbf{Z}_{2}$} & $4 \mathrm{aa}$ & Conventional $\mathbf{Z}_{2}$ vertices \\
\hline & $4 b$ & Unconventional $\mathbf{Z}_{2}$ vertices \\
\hline & 5 & Model 1 \\
\hline & 6 & Model 2 \\
\hline & 7 & Model 3 \\
\hline & 8 & Model 4 \\
\hline \multirow[t]{3}{*}{$\mathbf{Z}_{3}$} & 9 & Conventional $\mathbf{Z}_{3}$ vertices \\
\hline & 10 & Model 5 \\
\hline & 11 & Model 6 \\
\hline \multirow[t]{3}{*}{$\mathbf{Z}_{4}$} & 12 & Conventional $\mathbf{Z}_{4}$ vertices \\
\hline & 13 & Model 7 \\
\hline & 14 & Model 8 \\
\hline \multirow[t]{4}{*}{$\mathbf{Z}_{6}$} & 15 & Conventional $\mathbf{Z}_{6}$ vertices \\
\hline & 16 & Model 9 \\
\hline & 17 & Model 10 \\
\hline & 18 & Model 11 \\
\hline
\end{tabular}

Table 3: A Table of Figures 

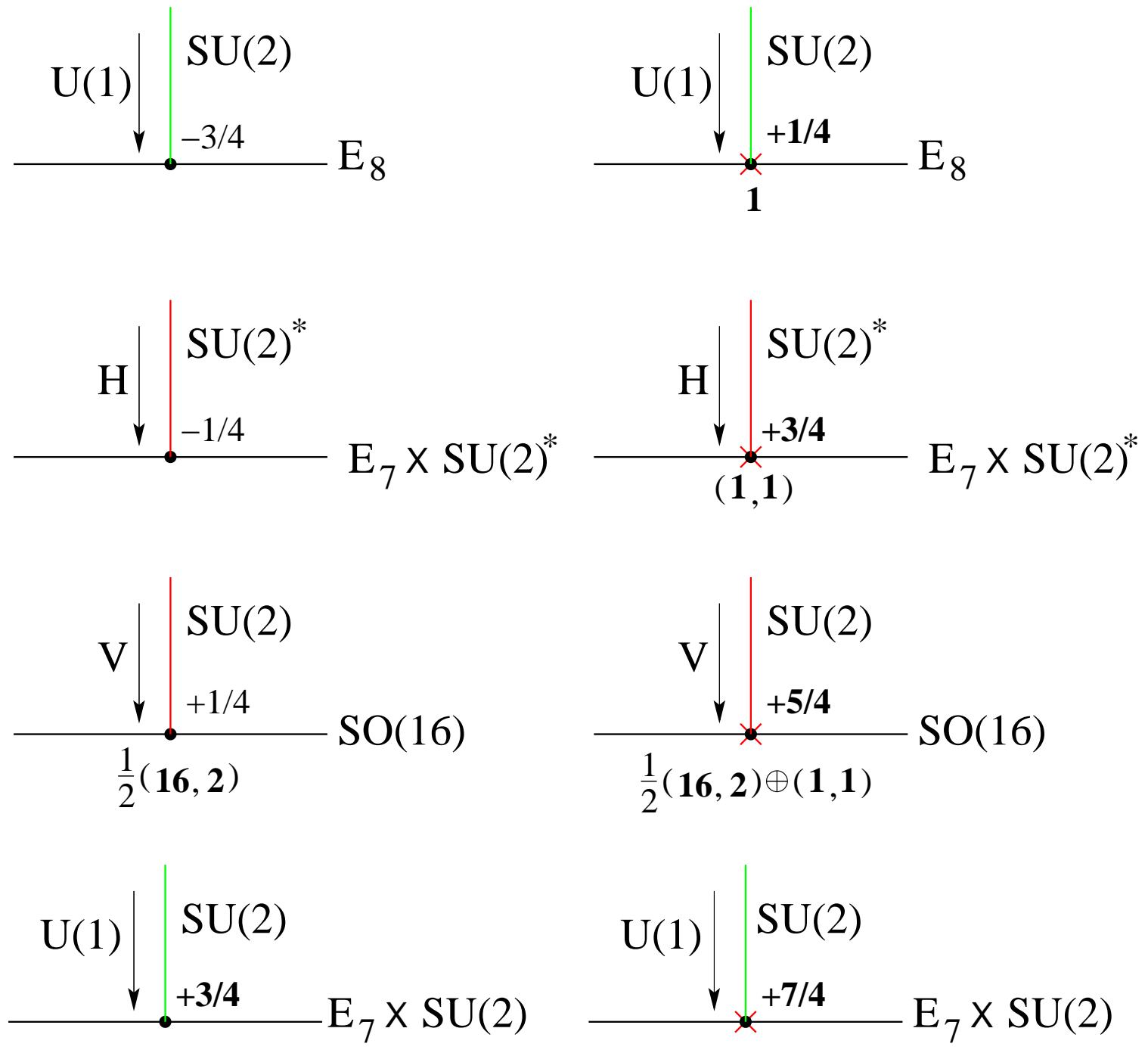

Figure 4a: Conventional primary vertices in the $\mathbf{Z}_{2}$ orbifold. These vertices have $S U(2)$ gauge group on the seven-planes. The asterix indicates identification of factors. 

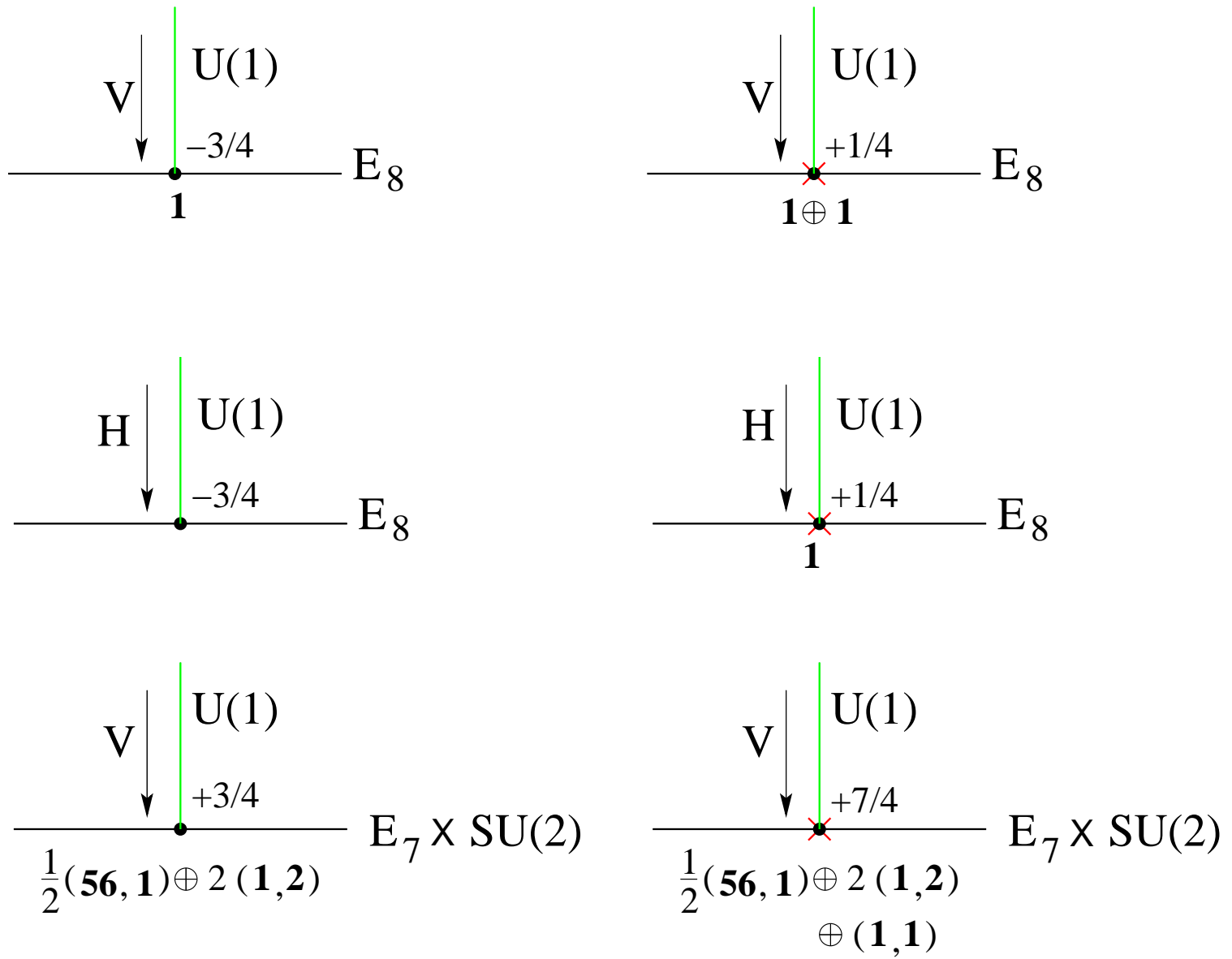

Figure 4b: Unconventional primary vertices in the $\mathbf{Z}_{2}$ orbifold. These vertices have $U(1)$ gauge group on the seven-planes. 


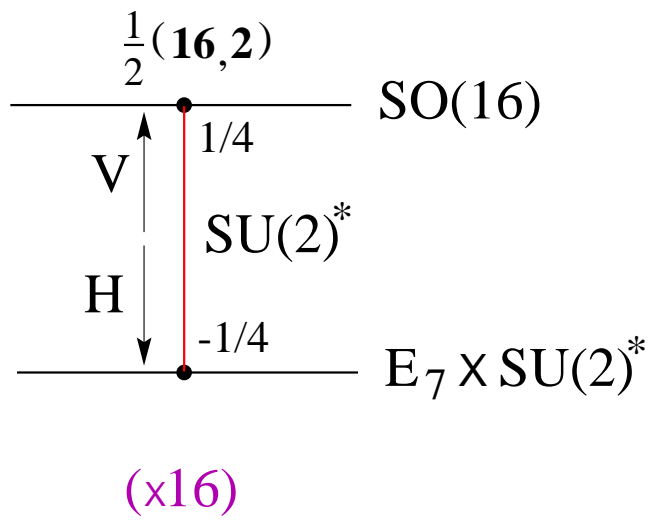

Figure 5: Global picture of Model 1, which has gauge group $E_{7} \times$ $S O(16) \times S U(2)$. This model has one tensor multiplet, 256 Vector multiplets transforming as $(\mathbf{1 3 3}, \mathbf{1}, \mathbf{1}) \oplus(\mathbf{1}, \mathbf{1 2 0}, \mathbf{1}) \oplus(\mathbf{1}, \mathbf{1}, \mathbf{3})$ and 500 hypermultiplets transforming as shown in Table 4.

\begin{tabular}{|c||c||c||c|}
\hline \multicolumn{4}{|c|}{500 Hypers } \\
\hline \hline \multirow{4}{*}{ Untwisted } & Sugra & $4(\mathbf{1}, \mathbf{1}, \mathbf{1})$ & 4 \\
& $M_{10}^{\text {top }}$ & $(\mathbf{1}, \mathbf{1 2 8}, \mathbf{1})$ & 128 \\
& $M_{10}^{\text {bottom }}$ & $(\mathbf{5 6 , 1}, \mathbf{2})$ & 112 \\
\hline & $M_{6}^{\text {top }}$ & $16 \times \frac{1}{2}(\mathbf{1}, \mathbf{1 6}, \mathbf{2})$ & 256 \\
& $M_{6}^{\text {bottom }}$ & & \\
& $M_{7}$ & & \\
\hline
\end{tabular}

Table 4: The hypermultiplet content of Model 1, in terms of $E_{7} \times S O(16) \times S U(2)$ representations. 


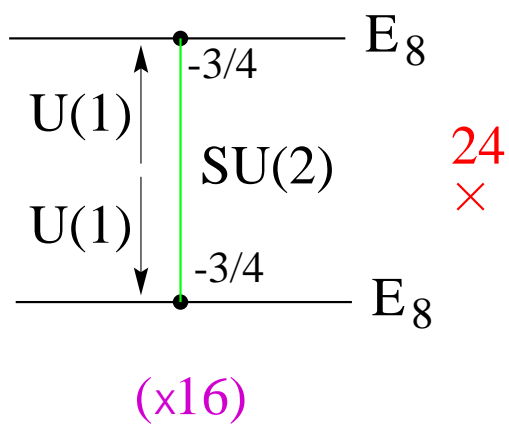

Figure 6: Global picture of Model 2, which has gauge group $E_{8} \times$ $E_{8} \times U(1)^{16}$ and 24 fivebranes. This model has 25 tensor multiplets (including 24 from the fivebranes), 512 vector multiplets transforming as $(\mathbf{2 4 8}, \mathbf{1}) \oplus(\mathbf{1}, \mathbf{2 4 8}) \oplus 16(\mathbf{1}, \mathbf{1})$ and 60 hypermultiplets transforming as shown in Table 5 .

\begin{tabular}{|c||c||c||c|}
\hline \multicolumn{3}{|c|}{60 Hypers } \\
\hline \hline \multirow{4}{*}{ Untwisted } & Sugra & $4(\mathbf{1}, \mathbf{1})$ & 4 \\
& $M_{10}^{\text {top }}$ & & \\
& $M_{10}^{\text {bottom }}$ & & \\
& $M_{6}^{\text {top }}$ & & \\
Twisted $_{1}$ & $M_{6}^{\text {bottom }}$ & & \\
& $M_{7}$ & $16 \times 2(\mathbf{1}, \mathbf{1})$ & 32 \\
\hline Fivebranes & & $24(\mathbf{1}, \mathbf{1})$ & 24 \\
\hline
\end{tabular}

Table 5: The hypermultiplet content of Model 2, in terms of $E_{8} \times E_{8}$ representations. 


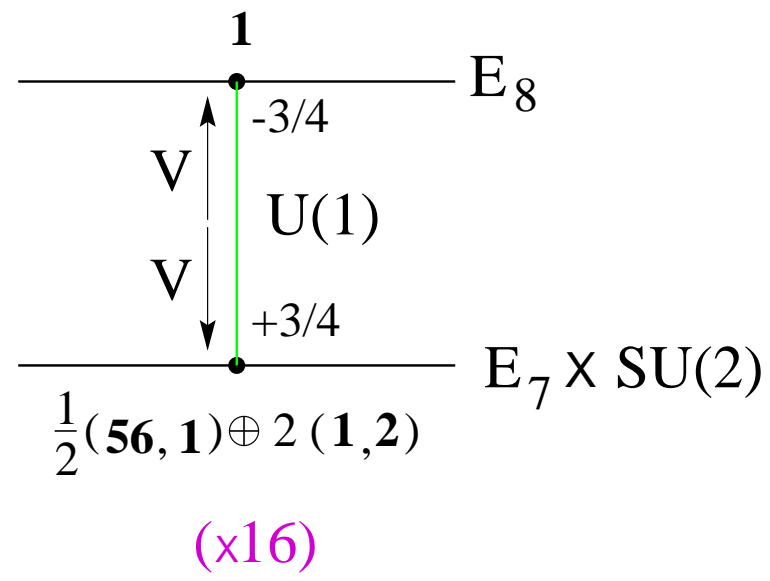

Figure 7: Global picture of Model 3, which has gauge group $E_{8} \times E_{7} \times$ $S U(2) \times U(1)^{16}$. This model has one untwisted tensor multiplet, 400 vector multiplets transforming as $(\mathbf{2 4 8}, \mathbf{1}, \mathbf{1}) \oplus(\mathbf{1}, \mathbf{1 3 3}, \mathbf{1}) \oplus(\mathbf{1}, \mathbf{1}, \mathbf{3}) \oplus$ $16(\mathbf{1}, \mathbf{1}, \mathbf{1})$, and 644 hypermultiplets transforming as shown in Table 6.

\begin{tabular}{|c||c||c||c|}
\hline \multicolumn{3}{|c|}{644 Hypers } \\
\hline \hline \multirow{3}{*}{ Untwisted } & Sugra & $4(\mathbf{1}, \mathbf{1})$ & 4 \\
& $M_{10}^{\text {top }}$ & $(\mathbf{1}, \mathbf{5 6}, \mathbf{2})$ & 112 \\
& $M_{10}^{\text {bottom }}$ & $16 \times(\mathbf{1}, \mathbf{1}, \mathbf{1})$ & 16 \\
& $M_{6}^{\text {top }}$ & $16 \times\left[\frac{1}{2}(\mathbf{1}, \mathbf{5 6}, \mathbf{1}) \oplus 2(\mathbf{1}, \mathbf{1}, \mathbf{2})\right]$ & 512 \\
Twisted $_{1}$ & $M_{6}^{\text {bottom }}$ & & \\
& $M_{7}$ & & \\
& & & \\
\hline
\end{tabular}

Table 6: The hypermultiplet content of Model 3 in terms of $E_{8} \times E_{7} \times S U(2)$ representations. 


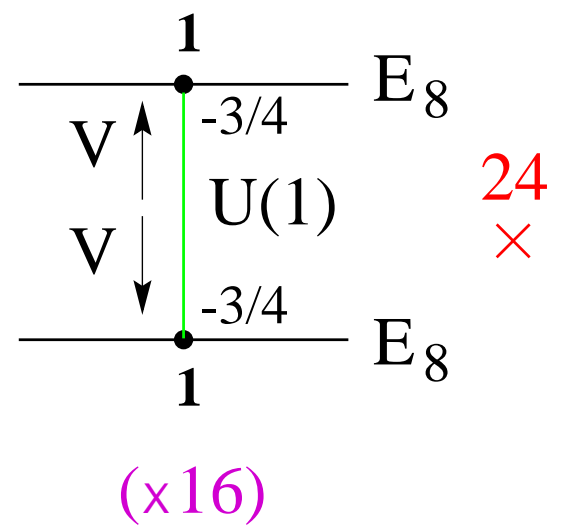

Figure 8: Global picture of Model 4, which has gauge group $E_{8} \times$ $E_{8} \times U(1)^{16}$. This model has 25 tensor multiplets (including 24 from the fivebranes) and 512 vector multiplets transforming as $(\mathbf{2 4 8}, \mathbf{1}) \oplus$ $(\mathbf{1}, \mathbf{2 4 8}) \oplus 16(\mathbf{1}, \mathbf{1})$, and 60 hypermultiplets transforming as shown in Table 7 .

\begin{tabular}{|c||c||c||c|}
\hline \multicolumn{3}{|c|}{60 Hypers } \\
\hline \hline \multirow{4}{*}{ Untwisted } & Sugra & $4(\mathbf{1}, \mathbf{1})$ & 4 \\
& $M_{10}^{\text {top }}$ & & \\
& $M_{10}^{\text {bottom }}$ & & \\
\hline & $M_{6}^{\text {top }}$ & $16 \times(\mathbf{1}, \mathbf{1})$ & 16 \\
Twisted $_{1}$ & $M_{6}^{\text {bottom }}$ & $16 \times(\mathbf{1}, \mathbf{1})$ & 16 \\
& $M_{7}$ & & \\
\hline Fivebranes & & $24 \times(\mathbf{1}, \mathbf{1})$ & 24 \\
\hline
\end{tabular}

Table 7: The hypermultiplet content of Model 4 in terms of $E_{8} \times E_{8}$ representations. 

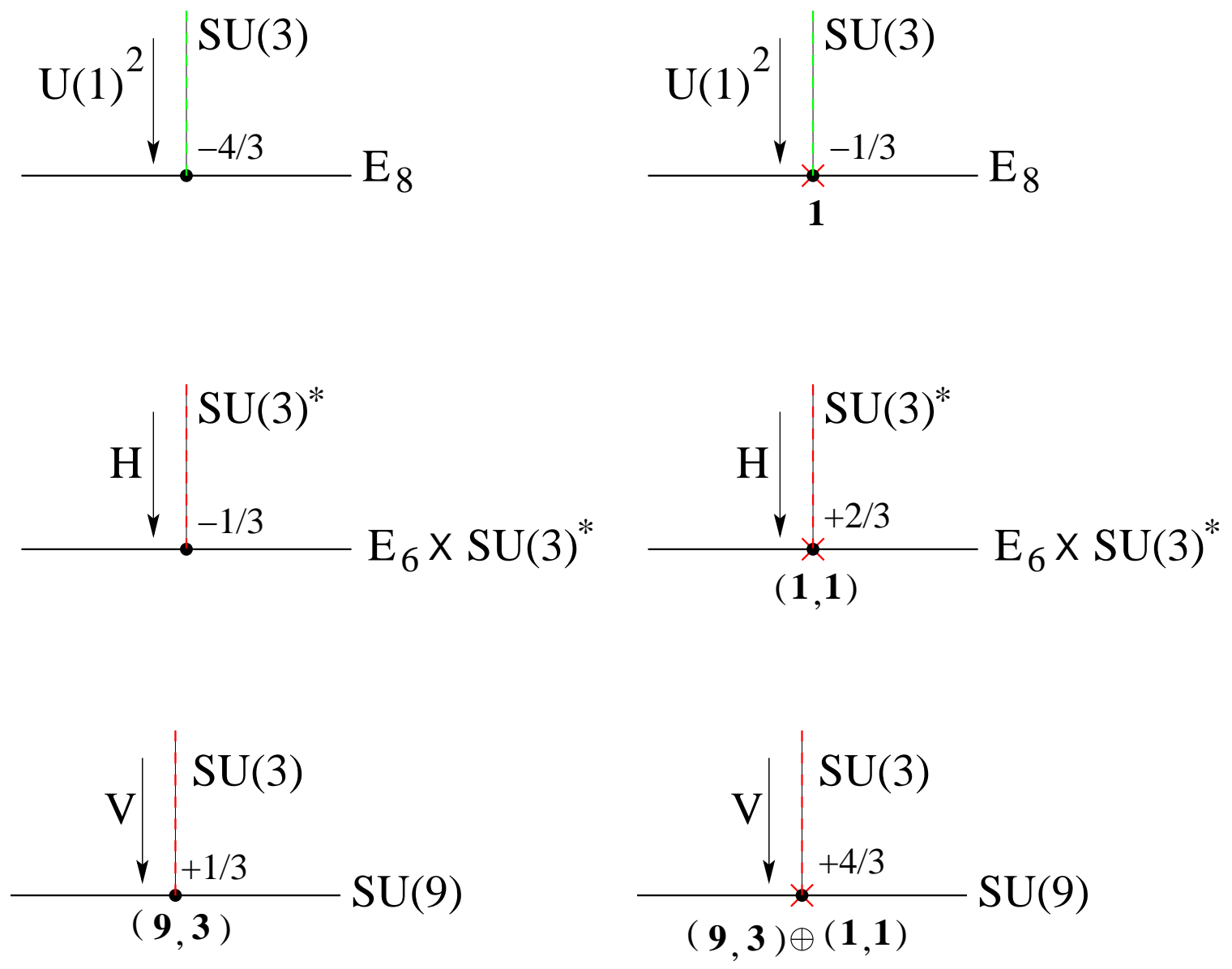

Figure 9: Consistent primary vertices in the $\mathbf{Z}_{3}$ orbifold 


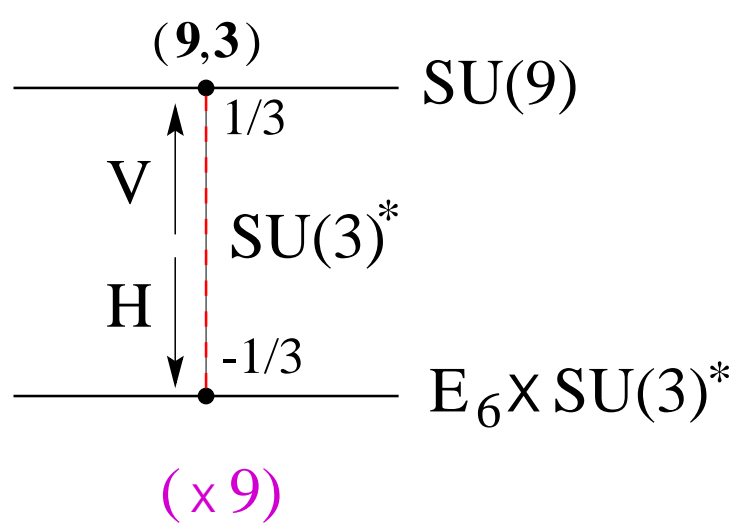

Figure 10: Global picture of Model 5, which has $6 D$ gauge group $E_{6} \times$ $S U(9) \times S U(3)$. This model has one untwisted tensor multiplet, 166 vector multiplets transforming as $(\mathbf{7 8}, \mathbf{1}, \mathbf{1}) \oplus(\mathbf{1}, \mathbf{8 0}, \mathbf{1}) \oplus(\mathbf{1}, \mathbf{1}, \mathbf{8})$, and 410 hypermultiplets transforming as shown in Table 8.

\begin{tabular}{|c||c||c||c|}
\hline \multicolumn{4}{|c|}{ 410 Hypers } \\
\hline \hline \multirow{4}{*}{ Untwisted } & Sugra & $2(\mathbf{1}, \mathbf{1}, \mathbf{1})$ & 2 \\
& $M_{10}^{\text {top }}$ & $(\mathbf{1}, \mathbf{8 4}, \mathbf{1})$ & 84 \\
& $M_{10}^{\text {bottom }}$ & $(\mathbf{2 7 , 1}, \mathbf{3})$ & 81 \\
\hline & top & $9 \times(\mathbf{1}, \mathbf{9}, \mathbf{3})$ & 243 \\
Twisted $_{1}$ & bottom & & \\
& $M_{7}$ & & \\
\hline
\end{tabular}

Table 8: The hypermultiplet content of Model 5 in terms of $E_{6} \times S U(9) \times S U(3)$ representations. 


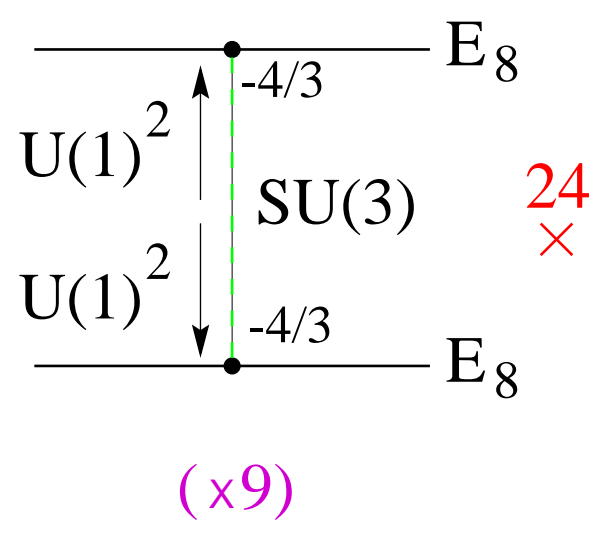

Figure 11: Global picture of Model 6, which has gauge group $E_{8} \times E_{8} \times$ $U(1)^{18}$ and 24 fivebranes. This model has 25 tensor multiplets (including 24 from the fivebranes) and 514 vector multiplets transforming as $(\mathbf{2 4 8}, \mathbf{1}) \oplus(\mathbf{1}, \mathbf{2 4 8}) \oplus 18(\mathbf{1}, \mathbf{1})$, and 62 hypermultiplets transforming as shown in Table 9.

\begin{tabular}{|c||c||c||c|}
\hline \multicolumn{4}{|c|}{62 Hypers } \\
\hline \hline \multirow{4}{*}{ Untwisted } & Sugra & $2(\mathbf{1}, \mathbf{1}, \mathbf{1})$ & 2 \\
& $M_{10}^{\text {top }}$ & & \\
& $M_{10}^{\text {bottom }}$ & & \\
\hline & top & & \\
Twisted $_{1}$ & bottom & & \\
& $M_{7}$ & $9 \times 4(\mathbf{1}, \mathbf{1}, \mathbf{1})$ & 36 \\
\hline Fivebranes & & $24(\mathbf{1}, \mathbf{1}, \mathbf{1})$ & 24 \\
\hline
\end{tabular}

Table 9: The hypermultiplet content of Model 6 in terms of $E_{8} \times E_{8}$ representations. 

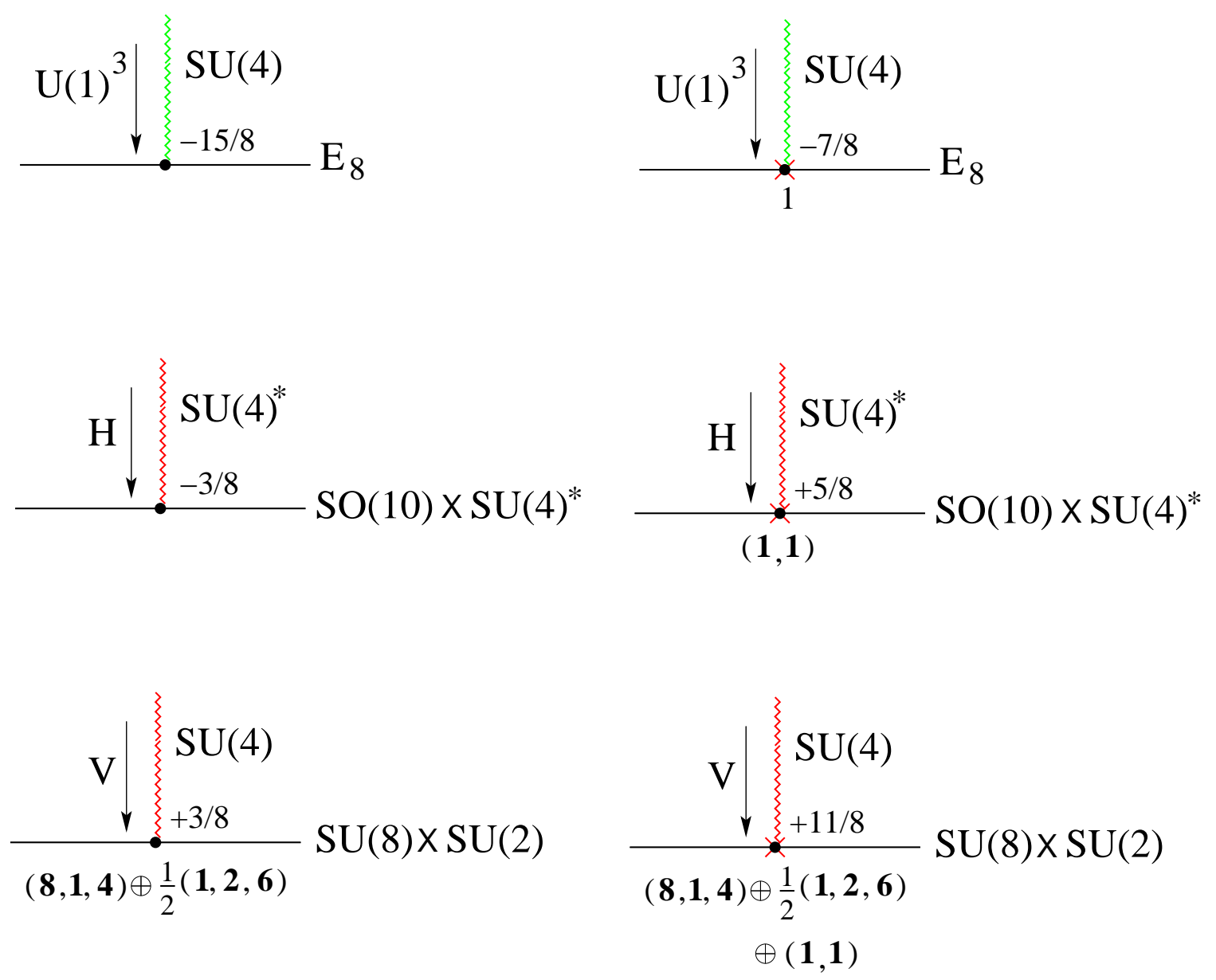

Figure 12: Consistent primary vertices in the $\mathbf{Z}_{4}$ orbifold 
$\mathrm{SU}(8) \times \mathrm{SU}(2) \quad \mathrm{E}_{7} \times \mathrm{SU}(2)$

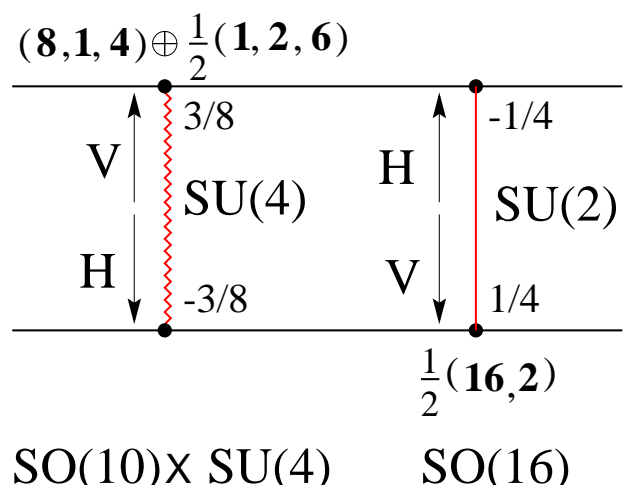

$(\times 4)$

Figure 13: Global picture of Model 7, which has gauge group $S O(10) \times$ $S U(8) \times S U(4) \times S U(2)$. This model has one untwisted tensor multiplet, 126 vector multiplets transforming as $(\mathbf{4 5}, \mathbf{1}, \mathbf{1}, \mathbf{1}) \oplus(\mathbf{1}, \mathbf{6 3}, \mathbf{1}, \mathbf{1}) \oplus$ $(\mathbf{1}, \mathbf{1}, \mathbf{1 5}, \mathbf{1}) \oplus(\mathbf{1}, \mathbf{1}, \mathbf{1}, \mathbf{3})$, and 370 hypermultiplets transforming as shown in Table 10.

\begin{tabular}{|c|c|c|c|}
\hline \multicolumn{4}{|c|}{370 Hypers } \\
\hline Untwisted & $\begin{array}{c}\text { Sugra } \\
M_{10}^{\text {top }} \\
M_{10}^{\text {bottom }}\end{array}$ & $\begin{array}{l}2(\mathbf{1}, \mathbf{1}, \mathbf{1}, \mathbf{1}) \\
(\mathbf{1}, \mathbf{2 8}, \mathbf{1}, \mathbf{2}) \\
(\mathbf{1 6}, \mathbf{1}, \mathbf{4}, \mathbf{1})\end{array}$ & $\begin{array}{c}2 \\
56 \\
64\end{array}$ \\
\hline Twisted $_{1}$ & $\begin{array}{c}\text { top } \\
\text { bottom } \\
M_{7}\end{array}$ & $4 \times\left[(\mathbf{1}, \mathbf{8}, \mathbf{1}, \mathbf{4}) \oplus \frac{1}{2}(\mathbf{1}, \mathbf{1}, \mathbf{2}, \mathbf{6})\right]$ & 152 \\
\hline Twisted $_{2}$ & $\begin{array}{c}\text { top } \\
\text { bottom } \\
M_{7}\end{array}$ & $6 \times\left[\frac{1}{2}(\mathbf{1 0}, \mathbf{1}, \mathbf{1}, \mathbf{2}) \oplus \frac{1}{2}(\mathbf{1}, \mathbf{1}, \mathbf{6}, \mathbf{2})\right]$ & 96 \\
\hline
\end{tabular}

Table 10: The hypermultiplet content of Model 7 in terms of $S O(10) \times S U(8) \times S U(4) \times S U(2)$ representations. 


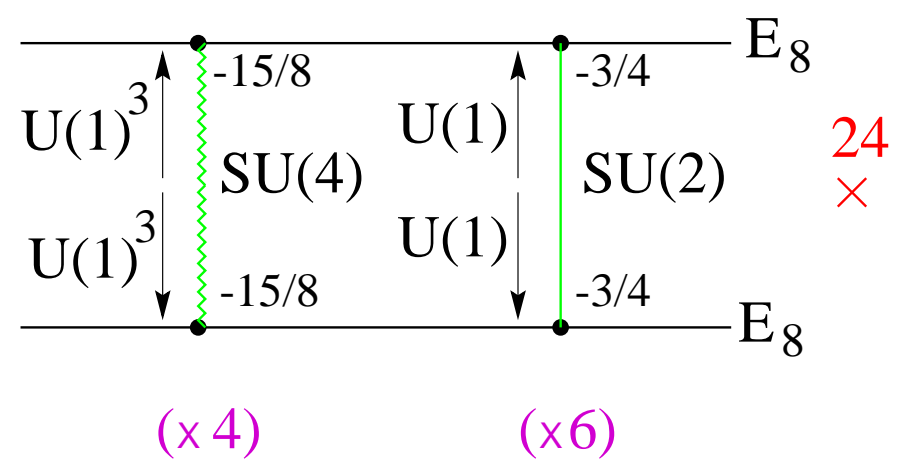

Figure 14: Global picture of Model 8, which has gauge group $E_{8} \times$ $E_{8} \times U(1)^{18}$ and 24 fivebranes. This model has 25 tensor multiplets (including 24 from the fivebranes), 514 vector multiplets transforming as $(\mathbf{2 4 8}, \mathbf{1}) \oplus(\mathbf{1}, \mathbf{2 4 8}, \mathbf{1}, \mathbf{1}) \oplus 18(\mathbf{1}, \mathbf{1})$, and 62 hypermultiplets transforming as shown in Table 11.

\begin{tabular}{|c|c|c|c|}
\hline \multicolumn{4}{|c|}{62 Hypers } \\
\hline Untwisted & $\begin{array}{c}\text { Sugra } \\
M_{10}^{\text {top }} \\
M_{10}^{\text {bottom }}\end{array}$ & $2(\mathbf{1}, \mathbf{1}, \mathbf{1})$ & 2 \\
\hline Twisted $_{1}$ & $\begin{array}{c}\text { top } \\
\text { bottom } \\
M_{7}\end{array}$ & $4 \times 6(\mathbf{1}, \mathbf{1}, \mathbf{1})$ & 24 \\
\hline Twisted $_{2}$ & $\begin{array}{c}\text { top } \\
\text { bottom } \\
M_{7}\end{array}$ & $6 \times 2(\mathbf{1}, \mathbf{1}, \mathbf{1})$ & 12 \\
\hline Fivebranes & & $24(\mathbf{1}, \mathbf{1}, \mathbf{1})$ & 24 \\
\hline
\end{tabular}

Table 11: The hypermultiplet content of Model 8 in terms of $E_{8} \times E_{8}$ representations. 

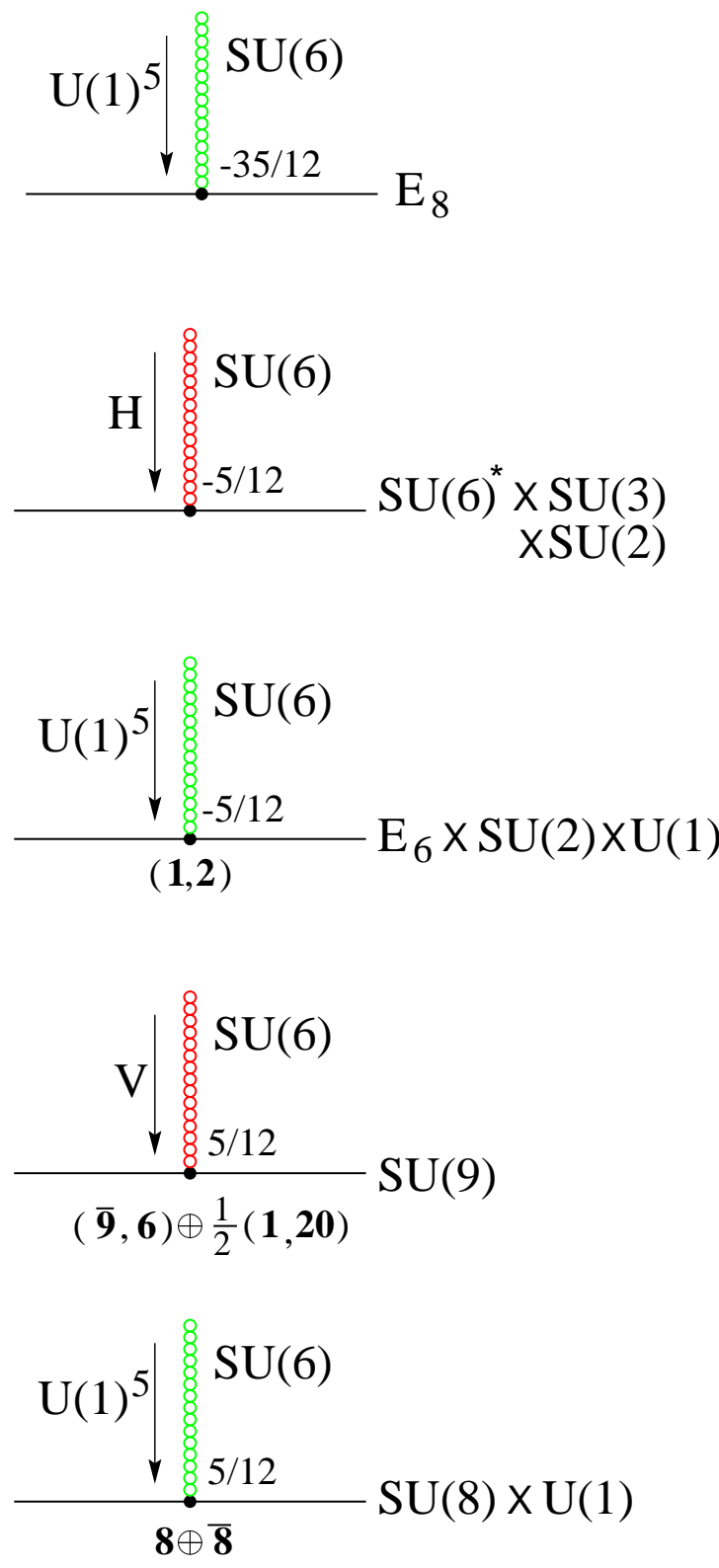
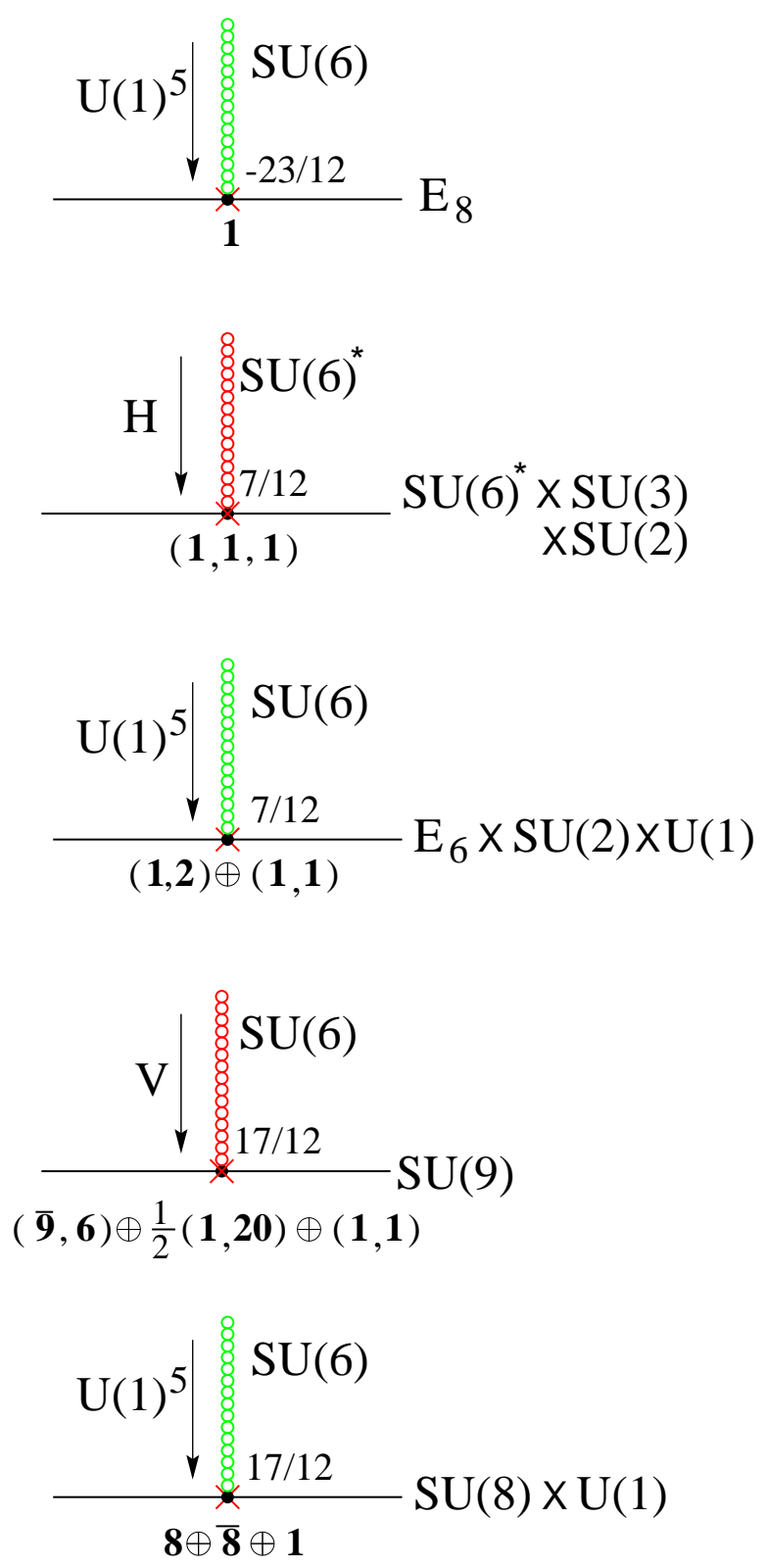

Figure 15: Consistent primary vertices in the $\mathbf{Z}_{6}$ orbifold 


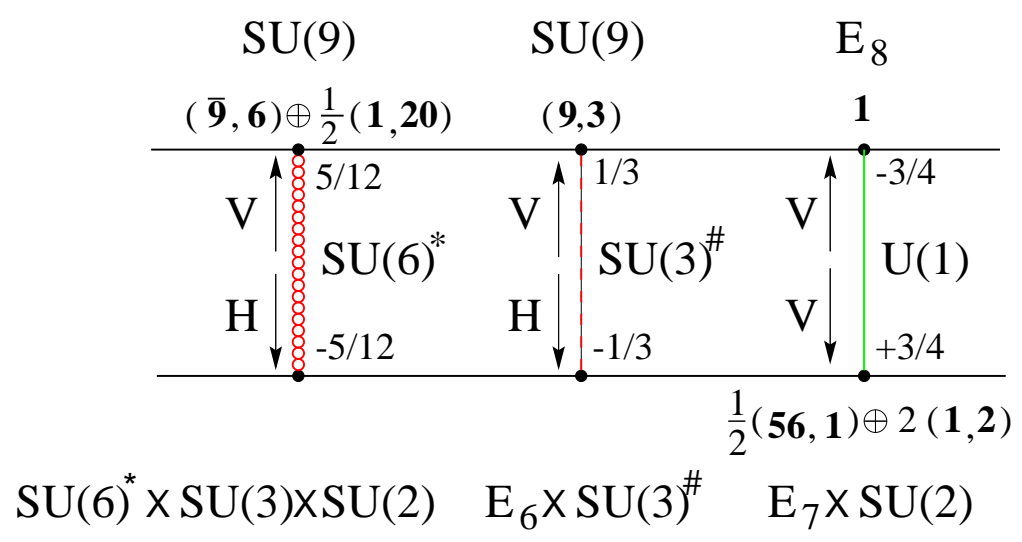

(1)

Figure 16: Global picture of Model 9, which has gauge group $S U(9) \times S U(6) \times S U(3) \times$ $S U(2) \times U(1)^{5}$. This model has one untwisted tensor multiplet, 131 vector multiplets transforming as $(\mathbf{8 0}, \mathbf{1}, \mathbf{1}, \mathbf{1}) \oplus(\mathbf{1}, \mathbf{3 5}, \mathbf{1}, \mathbf{1}) \oplus(\mathbf{1}, \mathbf{1}, \mathbf{8}, \mathbf{1}) \oplus(\mathbf{1}, \mathbf{1}, \mathbf{1}, \mathbf{3}) \oplus 5(\mathbf{1}, \mathbf{1}, \mathbf{1}, \mathbf{1})$, and 375 hypermultiplets transforming as shown in Table 12 .

\begin{tabular}{|c|c|c|c|}
\hline \multicolumn{4}{|c|}{375 Hypers } \\
\hline \multirow{3}{*}{ Untwisted } & Sugra & $2(\mathbf{1}, \mathbf{1}, \mathbf{1}, \mathbf{1})$ & 2 \\
\hline & $M_{10}^{\text {top }}$ & & \\
\hline & $M_{10}^{\text {bottom }}$ & $(1,6,3,2)$ & 36 \\
\hline \multirow{3}{*}{ Twisted $_{1}$} & top & $(\mathbf{9}, \mathbf{6}, \mathbf{1}, \mathbf{1}) \oplus \frac{1}{2}(\mathbf{1}, \mathbf{2 0}, \mathbf{1}, \mathbf{1})$ & 64 \\
\hline & bottom & & \\
\hline & $M_{7}$ & & \\
\hline \multirow{3}{*}{ Twisted $_{2}$} & top & $4 \times(\mathbf{9}, \mathbf{1}, \mathbf{3}, \mathbf{1})$ & 108 \\
\hline & bottom & & \\
\hline & $M_{7}$ & & \\
\hline \multirow{3}{*}{ Twisted $_{3}$} & top & $5 \times(\mathbf{1}, \mathbf{1}, \mathbf{1}, \mathbf{1})$ & 5 \\
\hline & bottom & $5 \times\left[(\mathbf{1}, \mathbf{6}, \mathbf{3}, \mathbf{1}) \oplus \frac{1}{2}(\mathbf{1}, \mathbf{2 0}, \mathbf{1}, \mathbf{1}) \oplus 2(\mathbf{1}, \mathbf{1}, \mathbf{1}, \mathbf{2})\right]$ & 160 \\
\hline & $M_{7}$ & & \\
\hline
\end{tabular}

Table 12: The hypermultiplet content of Model 9 in terms of $S U(9) \times S U(6) \times S U(3) \times S U(2)$ representations. 


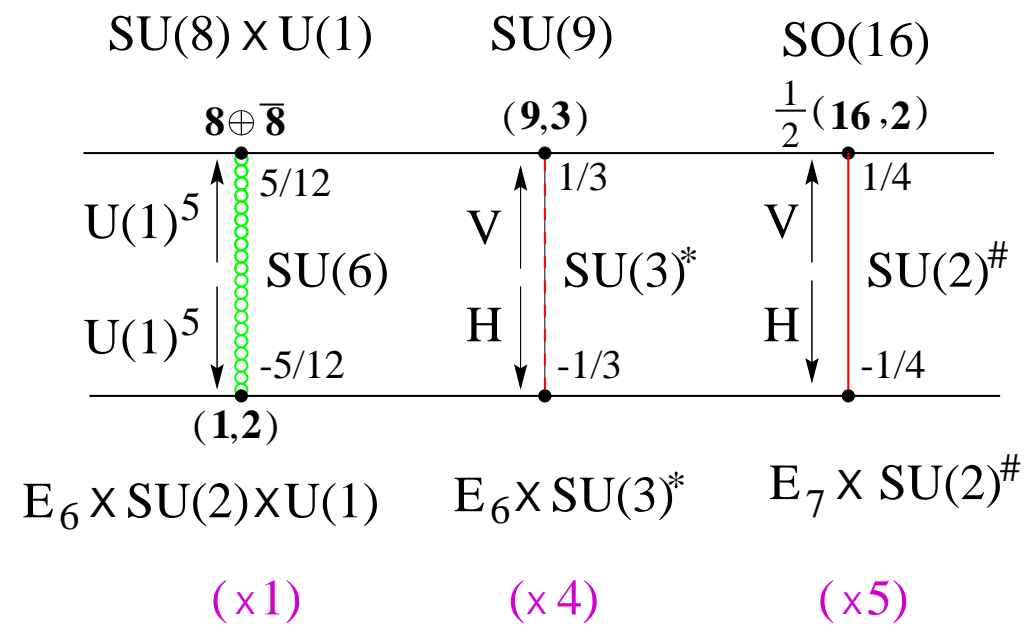

Figure 17: Global picture of Model 10, which has gauge group $E_{6} \times S U(8) \times S U(2) \times$ $U(1)^{7}$. This model has one untwisted tensor multiplet, 151 vector multiplets transforming as $(\mathbf{7 8}, \mathbf{1}, \mathbf{1}) \oplus(\mathbf{1}, \mathbf{6 3}, \mathbf{1}) \oplus(\mathbf{1}, \mathbf{1}, \mathbf{3}) \oplus 7(\mathbf{1}, \mathbf{1}, \mathbf{1})$, and 395 hypermultiplets transforming as shown in Table 13.

\begin{tabular}{|c||c||c||c|}
\hline \multicolumn{3}{|c|}{395 Hypers } \\
\hline \hline \multirow{4}{*}{ Untwisted } & Sugra & $2(\mathbf{1}, \mathbf{1}, \mathbf{1})$ & 2 \\
& $M_{10}^{\text {top }}$ & $(\mathbf{1}, \mathbf{5 6}, \mathbf{1}) \oplus(\mathbf{1}, \mathbf{2 8}, \mathbf{1}) \oplus(\mathbf{1}, \mathbf{8}, \mathbf{1})$ & 92 \\
& $M_{10}^{\text {bottom }}$ & $(\mathbf{2 7}, \mathbf{1}, \mathbf{1}) \oplus(\mathbf{2 7}, \mathbf{1}, \mathbf{2}) \oplus 2(\mathbf{1}, \mathbf{1}, \mathbf{2})$ & 85 \\
\hline & top & $\mathbf{8} \oplus \overline{\mathbf{8}}$ & 16 \\
Twisted $_{1}$ & bottom & $(\mathbf{1}, \mathbf{1}, \mathbf{2})$ & 2 \\
& $M_{7}$ & $10(\mathbf{1}, \mathbf{1}, \mathbf{1})$ & 10 \\
\hline \multirow{4}{*}{ Twisted $_{2}$} & top & $4 \times[(\mathbf{1}, \mathbf{8}, \mathbf{3}) \oplus(\mathbf{1}, \mathbf{1}, \mathbf{3})]$ & 108 \\
& bottom & & \\
& $M_{7}$ & & \\
\hline & top & $5 \times \frac{1}{2}(\mathbf{1}, \mathbf{8}, \mathbf{2})$ & \\
Twisted $_{3}$ & bottom & & \\
& $M_{7}$ & & \\
\hline
\end{tabular}

Table 13: The hypermultiplet content of Model 10 in terms of $E_{6} \times S U(8) \times S U(2)$ representations. 


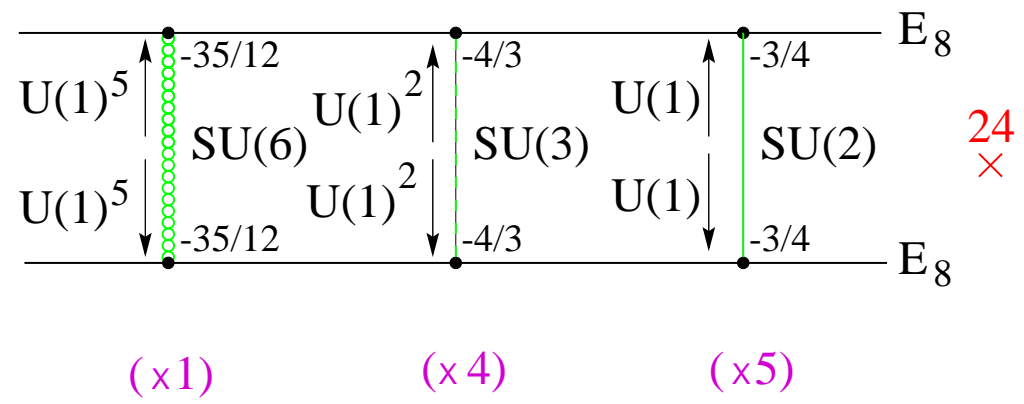

Figure 18: Global picture of Model 11, which has gauge group $E_{8} \times$ $E_{8} \times U(1)^{18}$ and 24 fivebranes. This model has 25 tensor multiplets (including 24 from the fivebranes), 514 vector multiplets transforming as $(\mathbf{2 4 8}, \mathbf{1}) \oplus(\mathbf{1}, \mathbf{2 4 8}) \oplus 18(\mathbf{1}, \mathbf{1})$, and 62 hypermultiplets transforming as shown in Table 14.

\begin{tabular}{|c|c|c|c|}
\hline \multicolumn{4}{|c|}{62 Hypers } \\
\hline Untwisted & $\begin{array}{l}\text { Sugra } \\
M_{10}^{\text {top }} \\
M_{10}^{\text {bottom }}\end{array}$ & $2(\mathbf{1}, \mathbf{1})$ & 2 \\
\hline Twisted $_{1}$ & $\begin{array}{c}\text { top } \\
\text { bottom } \\
M_{7}\end{array}$ & $1 \times 10(\mathbf{1}, \mathbf{1})$ & 10 \\
\hline Twisted $_{2}$ & $\begin{array}{c}\text { top } \\
\text { bottom } \\
M_{7}\end{array}$ & $4 \times 4(\mathbf{1}, \mathbf{1})$ & 16 \\
\hline Twisted $_{3}$ & $\begin{array}{c}\text { top } \\
\text { bottom } \\
M_{7}\end{array}$ & $5 \times 2(\mathbf{1}, \mathbf{1})$ & 10 \\
\hline Fivebranes & & $24(\mathbf{1}, \mathbf{1})$ & 24 \\
\hline
\end{tabular}

Table 14: The hypermultiplet content of Model 11 in terms of $E_{8} \times E_{8}$ representations. 


\section{Conclusions}

We have streamlined the technology developed in our previous papers [7, \& to enable us to determine $M$-theory orbifold anomalies soley in terms of rational data. This permits an algorithmic search for consistent orbifold vertices and allows us to complete twisted sector states, including those localized on certain odd-dimensional planes. We have explained a comprehensive set of models describing portions of the low-energy moduli space corresponding to $M$-theory compactified on $S^{1} / \mathbf{Z}_{2} \times K 3$ orbifolds, and discussed a number of technical and conceptual issues associated with these. This technology has been developed with the expressed intent of enabling a search for phenomenologically more interesting compactifications to four dimensions. We are currently investigating a large class of $T^{7} /\left(\mathbf{Z}_{M} \times \mathbf{Z}_{N}\right)$ and $T^{7} /\left(\mathbf{Z}_{M} \times \mathbf{Z}_{N} \times \mathbf{Z}_{L}\right)$ M-theory orbifolds, where the technol-

ogy described in this paper has a natural extension. Our aim is to partially delineate how nonperturbative string effects, particularly those involving fivebrane-mediated phase transitions can modify phenomenological predictions based on perturbative orbifolds, and facilitate the search for a more fundamental description of $M$-theory. This will be described in forthcoming papers.

\section{Appendix: Local Vertex Analyses}

This appendix contains tables listing the rational parameters associated with each of the consistent local vertices described in the main text. The data contained in these tables are sufficient to reconstruct the entire local anomaly for each vertex, including all gravitational, mixed and pure gauge terms, and to verify consistency using equation (4.4).

The method of analysis is as follows. For a specific vertex, we determine the $E_{8}$ branching rule to the desired subgroup. (These are tabulated in reference [15.) From this branching rule, it is straightforward to determine $V^{(n)}$ and $H^{(n)}$. The fixed input for a given choice of breaking pattern include the orbifold geometric parameters $f_{(n)}$ (which determine $f$ ) and the supergravity anomaly factor $P$, which is determined by the untwisted hypermultiplet multiplicities $h_{(n)}$, and the representation indices $\mathcal{I}_{4}, \mathcal{I}_{2,2}$ and $\mathcal{I}_{2}$ for each possible representation in the selected gauge group. All of this data, except for the representation indices, are also found in Table 1. Representation indices can be determined using the technology described in [16]. In the tables, we include the needed representation indices for easy verification purposes. These numbers represent the keys to the analysis, while equation(4.4) represents the lock. We encourage the reader to plug the data in the tables into (4.4) to show that each set solves all of the requirements. All 
of the parameters in the tables can be cross checked.

As an example, we will describe the analysis A.6 corresponding to the $\mathbf{Z}_{3}$ orbifold with branching $E_{8} \rightarrow E_{6} \times S U(3)$. The branching rule is $\mathbf{2 4 8} \rightarrow(\mathbf{7 8}, \mathbf{1}) \oplus(\mathbf{1}, \mathbf{8}) \oplus$ $(\mathbf{2 7}, \mathbf{3}) \oplus(\overline{\mathbf{2}}, \overline{\mathbf{3}})$. The adjoint representation of the broken subgroup corresponds to $78+8=86$ vector multiplets, so that $V^{(1)}=86$. The remaining terms in the decomposition correspond to $2(27 \times 3)=162$ hypermultiplets. However, half of these hypermultiplets are projected out by the $\mathbf{Z}_{3}$ projection, due to the nature of the $\mathbf{Z}_{3}$ action on the $E_{8}$ root lattice. Because of this, $H^{(1)}=27 \times 3=81$. Although it is possible to resolve projections such as this by constructing projection matrices 1 , it is relatively simple to resolve the effect of such projections by enforcing anomaly cancellation.

The parameters $n_{0}(\mathcal{R})$, for the four representations $\mathcal{R}$ given by the $\mathbf{7 8}$ and $\mathbf{2 7}$ of $E_{6}$ and the $\mathbf{8}$ and $\mathbf{3}$ of $S U(3)$, describe a straightforward decomposition of $E_{8}$ which is insensitive to the $\mathbf{Z}_{3}$ projection. As explained above, this is because the parameters $n_{0}(\mathcal{R})$ appears in the inflow anomaly, which involves only classical fields. As a result of this, the division by two reflected in $H^{(1)}$ is not reflected in $n_{0}(\mathcal{R})$. Thus, $n_{0}(\mathbf{2 7})=6$ rather than 3. On the other hand, the parameters $n_{1}(\mathcal{R})$ appear in the quantum anomaly, which $i s$ sensitive to the $\mathbf{Z}_{3}$ projection. Thus, the division by two $i s$ reflected in $n_{1}(\mathcal{R})$. (For instance $n_{1}(\mathbf{2 7})=-3$, since only three $\mathbf{2 7}$ hypermultiplets are $\mathbf{Z}_{3}$ invariant. Recall that hypermultiplets contribute with a minus sign in the quantum anomaly.) Analogous statements pertain to all of the tables included below.

\footnotetext{
${ }^{10}$ We avoid referring to "shift vectors" since it is not known what analog $M$-theory involves to replace vertex operators in the perturbative string.
} 
A.1 The $\mathrm{Z}_{2}$ orbifold with $E_{8} \rightarrow E_{8}$

\begin{tabular}{|cc||cc||cc|}
\hline$n$ & $\mathcal{G}_{n}$ & $f_{(n)}$ & $V^{(n)}$ & $H^{(n)}$ \\
\hline \hline 1 & $E_{8}$ & 16 & 248 & 0 \\
\hline
\end{tabular}

\begin{tabular}{|c||cc||c|}
\hline$f$ & $P$ & $Q$ & $P+Q$ \\
\hline \hline 16 & $\frac{15}{2}$ & $\frac{31}{2}$ & 23 \\
\hline
\end{tabular}

\begin{tabular}{|c||cc||ccc||ccc||c|}
\hline$I$ & $\mathcal{G}_{I}$ & $\mathcal{R}$ & $\mathcal{I}_{4}$ & $\mathcal{I}_{2,2}$ & $\mathcal{I}_{2}$ & $n_{0}$ & $n_{1}$ & $\tilde{n}$ & $\mathrm{Z}$ \\
\hline \hline 1 & $E_{8}$ & $\mathbf{2 4 8 ^ { * }}$ & 0 & 9 & 30 & 1 & 1 & 0 & $\frac{1}{16}$ \\
\hline
\end{tabular}

\begin{tabular}{|c||cccc||c|}
\hline$I$ & $X_{I}$ & $Z_{I}^{(4)}$ & $Z_{I}^{(2)}$ & $Z_{I}^{(2,2)}$ & $\rho_{I}$ \\
\hline \hline 1 & 1 & 0 & $\frac{15}{8}$ & $\frac{9}{16}$ & 0 \\
\hline
\end{tabular}

\begin{tabular}{|c||c|}
\hline$g$ & $\rho$ \\
\hline \hline$-\frac{3}{4}$ & 0 \\
\hline
\end{tabular}

$$
n_{H}-n_{V}=\frac{1}{2}
$$


A.2 The $\mathrm{Z}_{2}$ orbifold with $E_{8} \rightarrow E_{7} \times S U(2)$

\begin{tabular}{|cc||cc||c|}
\hline$n$ & $\mathcal{G}_{n}$ & $f_{(n)}$ & $V^{(n)}$ & $H^{(n)}$ \\
\hline \hline 1 & $E_{7} \times S U(2)$ & 16 & 136 & 112 \\
\hline
\end{tabular}

\begin{tabular}{|c||cc||c|}
\hline$f$ & $P$ & $Q$ & $P+Q$ \\
\hline \hline 16 & $\frac{15}{2}$ & $\frac{3}{2}$ & 9 \\
\hline
\end{tabular}

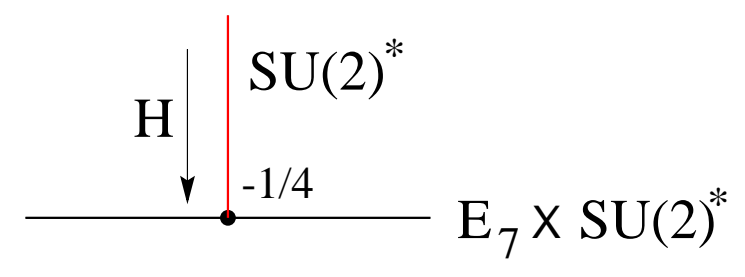

\begin{tabular}{|c||cc||ccc||ccc||c|}
\hline$I$ & $\mathcal{G}_{I}$ & $\mathcal{R}$ & $\mathcal{I}_{4}$ & $\mathcal{I}_{2,2}$ & $\mathcal{I}_{2}$ & $n_{0}$ & $n_{1}$ & $\tilde{n}$ & $\mathrm{Z}$ \\
\hline \hline 1 & $E_{7}$ & $\mathbf{1 3 3}^{*}$ & 0 & $\frac{1}{6}$ & 3 & 1 & 1 & 0 & $\frac{1}{16}$ \\
& & $\mathbf{5 6}$ & 0 & $\frac{1}{24}$ & 1 & 2 & -2 & 0 & $-\frac{1}{8}$ \\
\hline 2 & $S U(2)$ & $\mathbf{3}^{*}$ & 0 & 8 & 4 & 1 & 1 & $-\frac{1}{2}$ & $\frac{1}{16}$ \\
& & $\mathbf{2}$ & 0 & $\frac{1}{2}$ & 1 & 56 & -56 & 0 & $-\frac{7}{2}$ \\
\hline
\end{tabular}

\begin{tabular}{|c||cccc||c|}
\hline$I$ & $X_{I}$ & $Z_{I}^{(4)}$ & $Z_{I}^{(2)}$ & $Z_{I}^{(2,2)}$ & $\rho_{I}$ \\
\hline \hline 1 & $1 / 6$ & 0 & $\frac{1}{16}$ & $\frac{1}{192}$ & 0 \\
2 & 2 & 0 & $-\frac{21}{4}$ & $-\frac{21}{4}$ & 1 \\
\hline
\end{tabular}

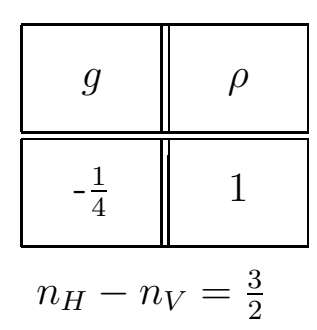


A.3 The $\mathbf{Z}_{2}$ orbifold with $E_{8} \rightarrow S O(16)$

\begin{tabular}{|cc||c||cc|}
\hline$n$ & $\mathcal{G}_{n}$ & $f_{(n)}$ & $V^{(n)}$ & $H^{(n)}$ \\
\hline \hline 1 & $S O(16)$ & 16 & 120 & 128 \\
\hline
\end{tabular}

\begin{tabular}{|c||cc||c|}
\hline$f$ & $P$ & $Q$ & $P+Q$ \\
\hline \hline 16 & $\frac{15}{2}$ & $-\frac{1}{2}$ & 7 \\
\hline
\end{tabular}

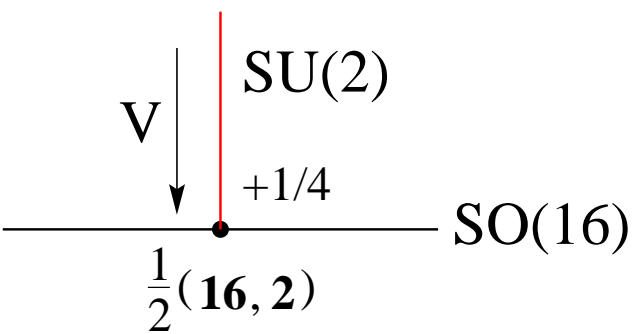

\begin{tabular}{|c||cc||ccc||ccc||c|}
\hline$I$ & $\mathcal{G}_{I}$ & $\mathcal{R}$ & $\mathcal{I}_{4}$ & $\mathcal{I}_{2,2}$ & $\mathcal{I}_{2}$ & $n_{0}$ & $n_{1}$ & $\tilde{n}$ & $\mathrm{Z}$ \\
\hline \hline 1 & $S O(16)$ & $\mathbf{1 2 8}$ & -8 & 6 & 16 & 1 & -1 & 0 & $-\frac{1}{16}$ \\
& $\mathbf{1 2 0 ^ { * }}$ & 8 & 3 & 14 & 1 & 1 & 0 & $\frac{1}{16}$ \\
& $\mathbf{1 6}$ & 1 & 0 & 1 & 0 & 0 & -1 & 0 \\
\hline 2 & $S U(2)$ & $\mathbf{3}^{*}$ & 0 & 8 & 4 & 0 & 0 & $\frac{1}{2}$ & 0 \\
& & $\mathbf{2}$ & 0 & $\frac{1}{2}$ & 1 & 0 & 0 & -8 & 0 \\
\hline
\end{tabular}

\begin{tabular}{|c||cccc|c|}
\hline$I$ & $X_{I}$ & $Z_{I}^{(4)}$ & $Z_{I}^{(2)}$ & $Z_{I}^{(2,2)}$ & $\rho_{I}$ \\
\hline \hline 1 & 1 & 0 & $-\frac{9}{8}$ & $-\frac{3}{16}$ & 0 \\
2 & 0 & 0 & -6 & 0 & 1 \\
\hline
\end{tabular}

\begin{tabular}{|c||c|}
\hline$g$ & $\rho$ \\
\hline \hline$\frac{1}{4}$ & 1 \\
\hline$n_{H}-n_{V}=\frac{29}{2}$
\end{tabular}


A.4 The $\mathbf{Z}_{2}$ orbifold with $E_{8} \rightarrow E_{7} \times S U(2)$

\begin{tabular}{|cc||c||cc|}
\hline$n$ & $\mathcal{G}_{n}$ & $f_{(n)}$ & $V^{(n)}$ & $H^{(n)}$ \\
\hline \hline 1 & $E_{7} \times S U(2)$ & 16 & 136 & 112 \\
\hline
\end{tabular}

\begin{tabular}{|c||cc||c|}
\hline$f$ & $P$ & $Q$ & $P+Q$ \\
\hline \hline 16 & $\frac{15}{2}$ & $\frac{3}{2}$ & 9 \\
\hline
\end{tabular}

$$
\frac{\mathrm{V} \mid \mathrm{J}_{+3 / 4}^{\mathrm{U}(1)}}{\frac{1}{2}(\mathbf{5 6}, \mathbf{1}) \oplus 2(\mathbf{1}, \mathbf{2})} \mathrm{E}_{7} \times \mathrm{SU}(2)
$$

\begin{tabular}{|c||cc||ccc||ccc||c|}
\hline$I$ & $\mathcal{G}_{I}$ & $\mathcal{R}$ & $\mathcal{I}_{4}$ & $\mathcal{I}_{2,2}$ & $\mathcal{I}_{2}$ & $n_{0}$ & $n_{1}$ & $\tilde{n}$ & $\mathrm{Z}$ \\
\hline \hline 1 & $E_{7}$ & $\mathbf{1 3 3}^{*}$ & 0 & $\frac{1}{6}$ & 3 & 1 & 1 & 0 & $\frac{1}{16}$ \\
& & $\mathbf{5 6}^{*}$ & 0 & $\frac{1}{24}$ & 1 & 2 & -2 & $-\frac{1}{2}$ & $-\frac{1}{8}$ \\
\hline 2 & $S U(2)$ & $\mathbf{3}^{*}$ & 0 & 8 & 4 & 1 & 1 & 0 & $\frac{1}{16}$ \\
& & $\mathbf{2}$ & 0 & $\frac{1}{2}$ & 1 & 56 & -56 & -2 & $-\frac{7}{2}$ \\
\hline
\end{tabular}

\begin{tabular}{|c||cccc|c|}
\hline$I$ & $X_{I}$ & $Z_{I}^{(4)}$ & $Z_{I}^{(2)}$ & $Z_{I}^{(2,2)}$ & $\rho_{I}$ \\
\hline \hline 1 & $\frac{1}{6}$ & 0 & $-\frac{7}{16}$ & $-\frac{1}{64}$ & 0 \\
2 & 2 & 0 & $-\frac{21}{4}$ & $-\frac{9}{4}$ & 0 \\
\hline
\end{tabular}

\begin{tabular}{|c||c|}
\hline$g$ & $\rho$ \\
\hline \hline$\frac{3}{4}$ & 0 \\
\hline$n_{H}-n_{V}=\frac{63}{2}$
\end{tabular}


A.5 The $\mathbf{Z}_{3}$ orbifold with $E_{8} \rightarrow E_{8}$

\begin{tabular}{|cc||cc||cc|}
\hline$n$ & $\mathcal{G}_{n}$ & $f_{(n)}$ & $V^{(n)}$ & $H^{(n)}$ \\
\hline \hline 1 & $E_{8}$ & 9 & 248 & 0 \\
\hline
\end{tabular}

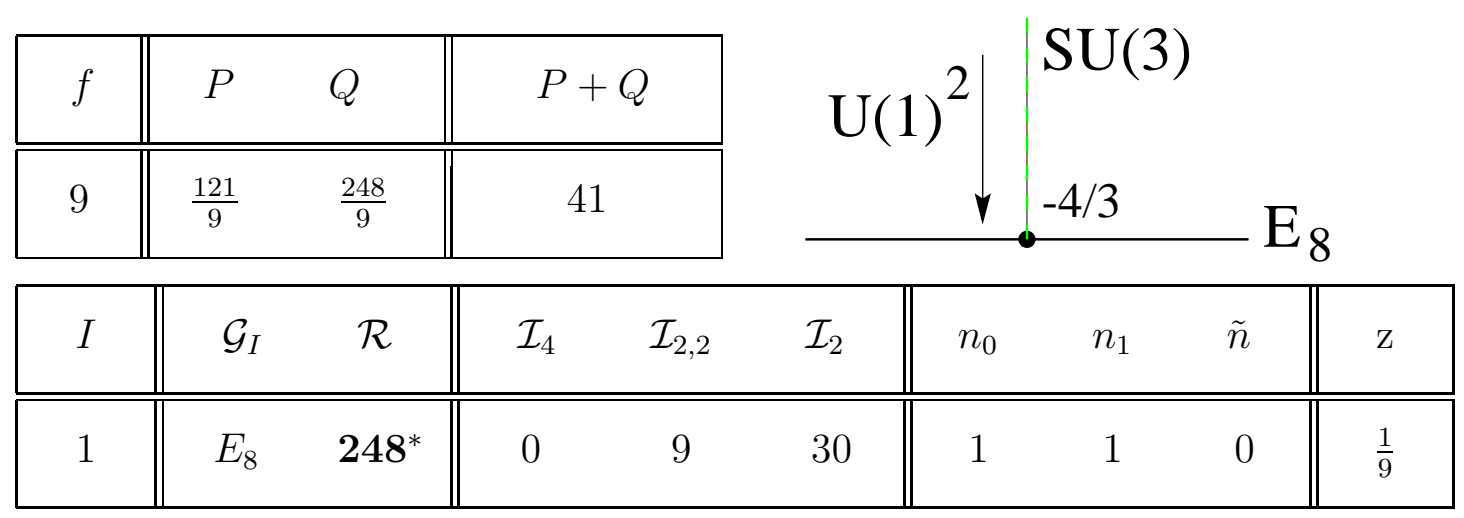

\begin{tabular}{|c||cccc||c|}
\hline$I$ & $X_{I}$ & $Z_{I}^{(4)}$ & $Z_{I}^{(2)}$ & $Z_{I}^{(2,2)}$ & $\rho_{I}$ \\
\hline \hline 1 & 1 & 0 & $\frac{10}{3}$ & 1 & 0 \\
\hline
\end{tabular}

\begin{tabular}{|c||c|}
\hline$g$ & $\rho$ \\
\hline \hline$-\frac{4}{3}$ & 0 \\
\hline
\end{tabular}

$$
n_{H}-n_{V}=1
$$


A.6 The $\mathbf{Z}_{3}$ orbifold with $E_{8} \rightarrow E_{6} \times S U(3)$

\begin{tabular}{|cc||cc||c|}
\hline$n$ & $\mathcal{G}_{n}$ & $f_{(n)}$ & $V^{(n)}$ & $H^{(n)}$ \\
\hline \hline 1 & $E_{6} \times S U(3)$ & 9 & 86 & 81 \\
\hline
\end{tabular}

\begin{tabular}{|c||cc||c|}
\hline$f$ & $P$ & $Q$ & $P+Q$ \\
\hline \hline 9 & $\frac{121}{9}$ & $\frac{5}{9}$ & 14 \\
\hline
\end{tabular}

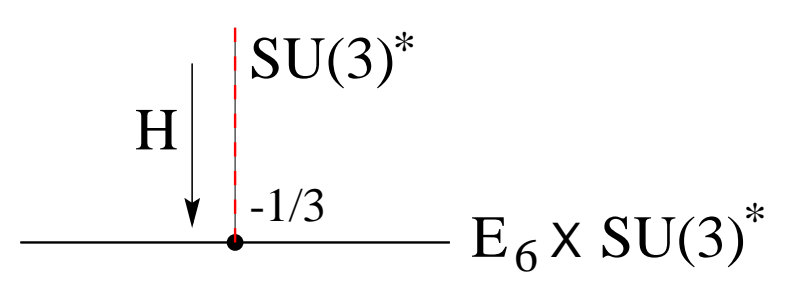

\begin{tabular}{|c||cc||ccc||ccc||c|}
\hline$I$ & $\mathcal{G}_{I}$ & $\mathcal{R}$ & $\mathcal{I}_{4}$ & $\mathcal{I}_{2,2}$ & $\mathcal{I}_{2}$ & $n_{0}$ & $n_{1}$ & $\tilde{n}$ & $\mathrm{Z}$ \\
\hline \hline 1 & $E_{6}$ & $\mathbf{7 8}^{*}$ & 0 & $\frac{1}{2}$ & 4 & 1 & 1 & 0 & $\frac{1}{9}$ \\
& & $\mathbf{2 7}$ & 0 & $\frac{1}{12}$ & 1 & 6 & -3 & 0 & $-\frac{1}{3}$ \\
\hline 2 & $S U(3)$ & $\mathbf{8}^{*}$ & 0 & 9 & 6 & 1 & 1 & $-\frac{1}{2}$ & $\frac{1}{9}$ \\
& & $\mathbf{3}$ & 0 & $\frac{1}{2}$ & 1 & 54 & -27 & 0 & -3 \\
\hline
\end{tabular}

\begin{tabular}{|c||cccc||c|}
\hline$I$ & $X_{I}$ & $Z_{I}^{(4)}$ & $Z_{I}^{(2)}$ & $Z_{I}^{(2,2)}$ & $\rho_{I}$ \\
\hline \hline 1 & $\frac{1}{3}$ & 0 & $\frac{1}{9}$ & $\frac{1}{36}$ & 0 \\
2 & 2 & 0 & $-\frac{16}{3}$ & -5 & 1 \\
\hline
\end{tabular}

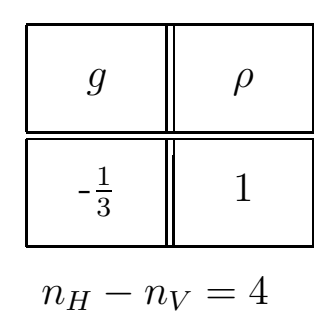


A.7 The $\mathbf{Z}_{3}$ orbifold with $E_{8} \rightarrow S U(9)$

\begin{tabular}{|cc||c||cc|}
\hline$n$ & $\mathcal{G}_{n}$ & $f_{(n)}$ & $V^{(n)}$ & $H^{(n)}$ \\
\hline \hline 1 & $S U(9)$ & 9 & 80 & 84 \\
\hline
\end{tabular}

\begin{tabular}{|c||cc||c|}
\hline$f$ & $P$ & $Q$ & $P+Q$ \\
\hline \hline 9 & $\frac{121}{9}$ & $-\frac{4}{9}$ & 13 \\
\hline
\end{tabular}

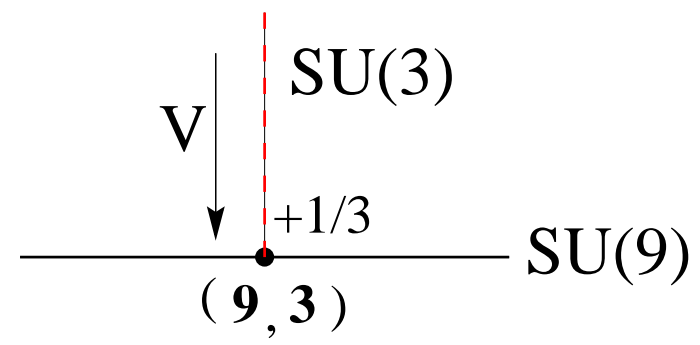

\begin{tabular}{|c||cc||ccc||ccc||c|}
\hline$I$ & $\mathcal{G}_{I}$ & $\mathcal{R}$ & $\mathcal{I}_{4}$ & $\mathcal{I}_{2,2}$ & $\mathcal{I}_{2}$ & $n_{0}$ & $n_{1}$ & $\tilde{n}$ & $\mathrm{Z}$ \\
\hline \hline 1 & $S U(9)$ & $\mathbf{8 4}$ & -9 & 15 & 21 & 2 & -1 & 0 & $-\frac{1}{9}$ \\
& $\mathbf{8 0 ^ { * }}$ & 18 & 6 & 18 & 1 & 1 & 0 & $\frac{1}{9}$ \\
& $\mathbf{9}$ & 1 & 0 & 1 & 0 & 0 & -3 & 0 \\
\hline 2 & $S U(3)$ & $\mathbf{8}^{*}$ & 0 & 9 & 6 & 0 & 0 & $\frac{1}{2}$ & 0 \\
& & & 0 & $\frac{1}{2}$ & 1 & 0 & 0 & -9 & 0 \\
\hline
\end{tabular}

\begin{tabular}{|c||cccc||c|}
\hline$I$ & $X_{I}$ & $Z_{I}^{(4)}$ & $Z_{I}^{(2)}$ & $Z_{I}^{(2,2)}$ & $\rho_{I}$ \\
\hline \hline 1 & 2 & 0 & $-\frac{10}{3}$ & -1 & 0 \\
2 & 0 & 0 & -6 & 0 & 1 \\
\hline
\end{tabular}

\begin{tabular}{|c||c|}
\hline$g$ & $\rho$ \\
\hline \hline$\frac{1}{3}$ & 1 \\
\hline
\end{tabular}


A.8 The $\mathrm{Z}_{4}$ orbifold with $E_{8} \rightarrow E_{8}$

\begin{tabular}{|cc||c||cc|}
\hline$n$ & $\mathcal{G}_{n}$ & $f_{(n)}$ & $V^{(n)}$ & $H^{(n)}$ \\
\hline \hline 1 & $E_{8}$ & 4 & 248 & 0 \\
2 & $E_{8}$ & $\frac{32}{3}$ & 248 & 0 \\
\hline
\end{tabular}

\begin{tabular}{|c|c|c|c|c|c|c|c|c|c|c|}
\hline$f$ & $P$ & $Q$ & \multicolumn{2}{|c|}{$P+Q$} & \multicolumn{2}{|c|}{$\mathrm{IJ}(1)^{3}$} & \multicolumn{4}{|c|}{ SU(4) } \\
\hline$\frac{32}{5}$ & 19 & $\frac{155}{4}$ & & & & & -15 & & & \\
\hline$I$ & $\mathcal{G}_{I}$ & $\mathcal{R}$ & $\mathcal{I}_{4}$ & $\mathcal{I}_{2,2}$ & $\mathcal{I}_{2}$ & $n_{0}$ & $n_{1}$ & $n_{2}$ & $\tilde{n}$ & z \\
\hline 1 & $E_{8}$ & $248^{*}$ & 0 & 9 & 30 & 1 & 1 & 1 & 0 & $\frac{5}{32}$ \\
\hline
\end{tabular}

\begin{tabular}{|c||cccc||c|}
\hline$I$ & $X_{I}$ & $Z_{I}^{(4)}$ & $Z_{I}^{(2)}$ & $Z_{I}^{(2,2)}$ & $\rho_{I}$ \\
\hline \hline 1 & 1 & 0 & $\frac{75}{16}$ & $\frac{45}{32}$ & 0 \\
\hline
\end{tabular}

\begin{tabular}{|c||c|}
\hline$g$ & $\rho$ \\
\hline \hline$-\frac{15}{8}$ & 0 \\
\hline
\end{tabular}

$$
n_{H}-n_{V}=\frac{3}{2}
$$


A.9 The $\mathbf{Z}_{4}$ orbifold with $E_{8} \rightarrow S O(10) \times S U(4)$

\begin{tabular}{|cc||c||cc|}
\hline$n$ & $\mathcal{G}_{n}$ & $f_{(n)}$ & $V^{(n)}$ & $H^{(n)}$ \\
\hline \hline 1 & $S O(10) \times S U(4)$ & 4 & 60 & 64 \\
2 & $S O(16)$ & $\frac{32}{3}$ & 120 & 128 \\
\hline
\end{tabular}

\begin{tabular}{|c||cc||c|}
\hline$f$ & $P$ & $Q$ & $P+Q$ \\
\hline \hline$\frac{32}{5}$ & 19 & $-\frac{1}{4}$ & $\frac{75}{4}$ \\
\hline
\end{tabular}

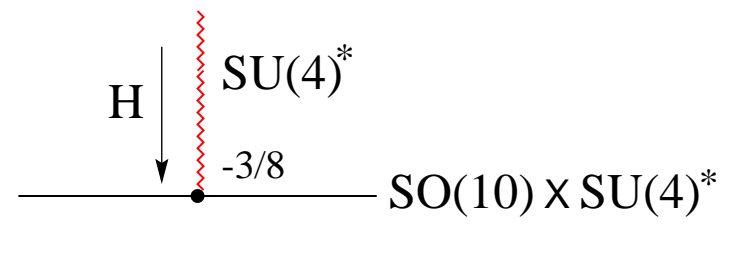

\begin{tabular}{|c||cc||ccc||cccc||c|}
\hline \multicolumn{1}{|||}{$I$} & $\mathcal{G}_{I}$ & $\mathcal{R}$ & $\mathcal{I}_{4}$ & $\mathcal{I}_{2,2}$ & $\mathcal{I}_{2}$ & $n_{0}$ & $n_{1}$ & $n_{2}$ & $\tilde{n}$ & $\mathrm{Z}$ \\
\hline \hline \multirow{2}{*}{1} & $S O(10)$ & $\mathbf{4 5}$ & 2 & 3 & 8 & 1 & 1 & 1 & 0 & $\frac{5}{32}$ \\
& $\mathbf{1 6}$ & -1 & $\frac{3}{4}$ & 2 & 8 & -4 & -8 & 0 & $-\frac{1}{4}$ \\
& $\mathbf{1 0}$ & 1 & 0 & 1 & 6 & 0 & 6 & 0 & $-\frac{9}{16}$ \\
\hline 2 & $S U(4)$ & $\mathbf{1 5}$ & 8 & 6 & 8 & 1 & 1 & 1 & $-\frac{1}{2}$ & $\frac{5}{32}$ \\
& & $\mathbf{6}$ & -4 & 3 & 2 & 10 & 0 & 10 & 0 & $-\frac{15}{16}$ \\
& $\mathbf{4}$ & 1 & 0 & 1 & 32 & -16 & -32 & 0 & -1 \\
\hline
\end{tabular}

\begin{tabular}{|c||cccc||c|}
\hline$I$ & $X_{I}$ & $Z_{I}^{(4)}$ & $Z_{I}^{(2)}$ & $Z_{I}^{(2,2)}$ & $\rho_{I}$ \\
\hline \hline 1 & 1 & 0 & $\frac{3}{16}$ & $\frac{9}{32}$ & 0 \\
2 & 2 & 0 & $-\frac{45}{8}$ & $-\frac{39}{8}$ & 1 \\
\hline
\end{tabular}

\begin{tabular}{|c||c|}
\hline$g$ & $\rho$ \\
\hline \hline$-\frac{3}{8}$ & 1 \\
\hline$n_{H}-n_{V}=\frac{15}{2}$
\end{tabular}


A.10 The $\mathrm{Z}_{4}$ orbifold with $E_{8} \rightarrow S U(8) \times S U(2)$

\begin{tabular}{|cc||c||cc|}
\hline$n$ & $\mathcal{G}_{n}$ & $f_{(n)}$ & $V^{(n)}$ & $H^{(n)}$ \\
\hline \hline 1 & $S U(8) \times S U(2)$ & 4 & 66 & 56 \\
2 & $E_{7} \times S U(2)$ & $\frac{32}{3}$ & 136 & 112 \\
\hline
\end{tabular}

\begin{tabular}{|c||cc||c|}
\hline$f$ & $P$ & $Q$ & $P+Q$ \\
\hline \hline$\frac{32}{5}$ & 19 & $\frac{1}{4}$ & $\frac{77}{4}$ \\
\hline
\end{tabular}

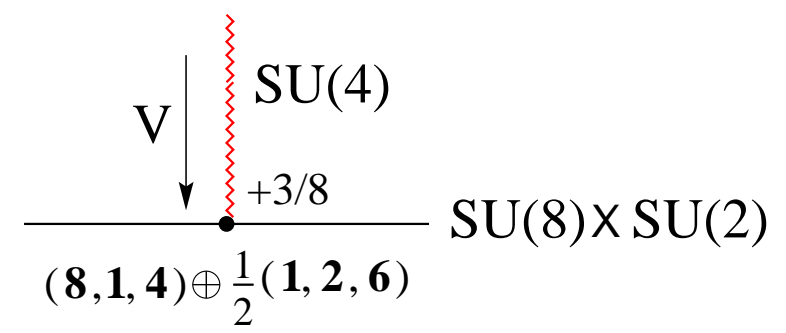

\begin{tabular}{|c|c|c|c|c|c|c|c|c|c|c|}
\hline$I$ & $\mathcal{G}_{I}$ & $\mathcal{R}$ & $\mathcal{I}_{4}$ & $\mathcal{I}_{2,2}$ & $\mathcal{I}_{2}$ & $n_{0}$ & $n_{1}$ & $n_{2}$ & $\tilde{n}$ & $\mathrm{Z}$ \\
\hline \multirow[t]{4}{*}{1} & $S U(8)$ & 70 & -16 & 18 & 20 & 1 & 0 & 1 & 0 & $-\frac{3}{32}$ \\
\hline & & $63^{*}$ & 16 & 6 & 16 & 1 & 1 & 1 & 0 & $\frac{5}{32}$ \\
\hline & & 28 & 0 & 3 & 6 & 4 & -2 & -4 & 0 & $-\frac{1}{8}$ \\
\hline & & 8 & 1 & 0 & 1 & 0 & 0 & 0 & -4 & 0 \\
\hline \multirow[t]{2}{*}{2} & $S U(2)$ & $3^{*}$ & 0 & 8 & 4 & 1 & 1 & 1 & 0 & $\frac{5}{32}$ \\
\hline & & 2 & 0 & $\frac{1}{2}$ & 1 & 56 & -28 & -56 & -3 & $-\frac{7}{4}$ \\
\hline \multirow[t]{3}{*}{3} & $S U(4)$ & $15^{*}$ & 8 & 6 & 8 & 0 & 0 & 0 & $\frac{1}{2}$ & 0 \\
\hline & & 6 & -4 & 3 & 2 & 0 & 0 & 0 & -1 & 0 \\
\hline & & 4 & 1 & 0 & 1 & 0 & 0 & 0 & -8 & 0 \\
\hline
\end{tabular}

\begin{tabular}{|c||cccc||c|}
\hline$I$ & $X_{I}$ & $Z_{I}^{(4)}$ & $Z_{I}^{(2)}$ & $Z_{I}^{(2,2)}$ & $\rho_{I}$ \\
\hline \hline 1 & 2 & 0 & $-\frac{33}{8}$ & $-\frac{9}{8}$ & 0 \\
2 & 2 & 0 & $-\frac{33}{8}$ & $-\frac{9}{8}$ & 0 \\
3 & 0 & 0 & -6 & 0 & 1 \\
\hline
\end{tabular}

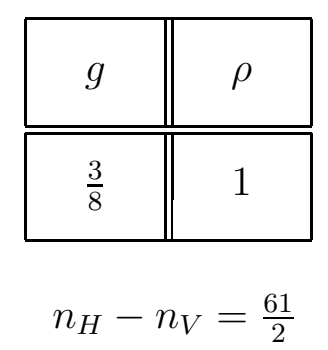




\section{A.11 The $\mathrm{Z}_{6}$ orbifold with $E_{8} \rightarrow E_{8}$}

\begin{tabular}{|cc||c||cc|}
\hline$n$ & $\mathcal{G}_{n}$ & $f_{(n)}$ & $V^{(n)}$ & $H^{(n)}$ \\
\hline \hline 1 & $E_{8}$ & 1 & 248 & 0 \\
2 & $E_{8}$ & $\frac{9}{4}$ & 248 & 0 \\
3 & $E_{8}$ & $\frac{16}{5}$ & 248 & 0 \\
\hline
\end{tabular}

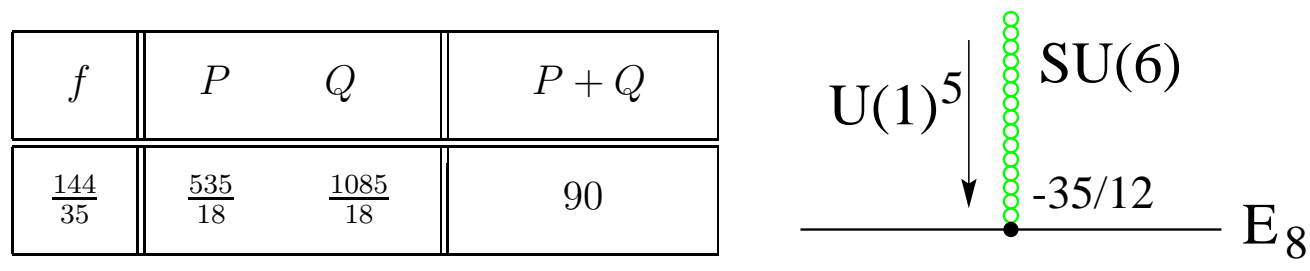

\begin{tabular}{|c||cc||ccc||ccccc||c|}
\hline$I$ & $\mathcal{G}_{I}$ & $\mathcal{R}$ & $\mathcal{I}_{4}$ & $\mathcal{I}_{2,2}$ & $\mathcal{I}_{2}$ & $n_{0}$ & $n_{1}$ & $n_{2}$ & $n_{3}$ & $\tilde{n}$ & $\mathrm{Z}$ \\
\hline \hline 1 & $E_{8}$ & $\mathbf{2 4 8}$ & 0 & 9 & 30 & 1 & 1 & 1 & 1 & 0 & $\frac{35}{144}$ \\
\hline
\end{tabular}

\begin{tabular}{|c||cccc||c|}
\hline$I$ & $X_{I}$ & $Z_{I}^{(4)}$ & $Z_{I}^{(2)}$ & $Z_{I}^{(2,2)}$ & $\rho_{I}$ \\
\hline \hline 1 & 1 & 0 & $\frac{175}{24}$ & $\frac{35}{16}$ & 0 \\
\hline
\end{tabular}

\begin{tabular}{|c||c|}
\hline$g$ & $\rho$ \\
\hline \hline$-\frac{35}{12}$ & 0 \\
\hline
\end{tabular}

$$
n_{H}-n_{V}=\frac{5}{2}
$$


A.12 The $\mathbf{Z}_{6}$ orbifold with $E_{8} \rightarrow S U(8) \times U(1)$

\begin{tabular}{|cc||c||cc|}
\hline$n$ & $\mathcal{G}_{n}$ & $f_{(n)}$ & $V^{(n)}$ & $H^{(n)}$ \\
\hline \hline 1 & $S U(8) \times U(1)$ & 1 & 64 & 92 \\
2 & $S U(9)$ & $\frac{9}{4}$ & 80 & 84 \\
3 & $S O(16)$ & $\frac{16}{5}$ & 120 & 128 \\
\hline
\end{tabular}

\begin{tabular}{|c|c|c|c|c|c|c|}
\hline$f$ & $P$ & $Q$ & $P+Q$ & \multirow[t]{2}{*}{$\mathrm{U}(1)^{5}$} & SU(6) & \\
\hline$\frac{144}{35}$ & $\frac{535}{18}$ & $-\frac{427}{18}$ & 6 & & $5 / 12$ & $\mathrm{SU}(8) \times \mathrm{U}(1)$ \\
\hline
\end{tabular}

\begin{tabular}{|c|c|c|c|c|c|c|c|c|c|c|c|}
\hline$I$ & $\mathcal{G}_{I}$ & $\mathcal{R}$ & $\mathcal{I}_{4}$ & $\mathcal{I}_{2,2}$ & $\mathcal{I}_{2}$ & $n_{0}$ & $n_{1}$ & $n_{2}$ & $n_{3}$ & $\tilde{n}$ & z \\
\hline \multirow[t]{4}{*}{1} & $S U(8)$ & $63^{*}$ & 16 & 6 & 16 & 1 & 1 & 1 & 1 & 0 & $\frac{35}{144}$ \\
\hline & & 56 & -9 & 12 & 15 & 2 & -1 & -1 & -2 & 0 & $\frac{5}{72}$ \\
\hline & & 28 & 0 & 3 & 6 & 2 & -1 & -1 & +2 & 0 & $-\frac{85}{72}$ \\
\hline & & 8 & 1 & 0 & 1 & 2 & -1 & +2 & -2 & -2 & $-\frac{91}{72}$ \\
\hline
\end{tabular}

\begin{tabular}{|c||cccc||c|}
\hline$I$ & $X_{I}$ & $Z_{I}^{(4)}$ & $Z_{I}^{(2)}$ & $Z_{I}^{(2,2)}$ & $\rho_{I}$ \\
\hline \hline 1 & 2 & 0 & $-\frac{65}{12}$ & $-\frac{5}{4}$ & 0 \\
\hline
\end{tabular}

\begin{tabular}{|c||c|}
\hline$g$ & $\rho$ \\
\hline \hline$\frac{5}{12}$ & 0 \\
\hline
\end{tabular}

$$
n_{H}-n_{V}=\frac{37}{2}
$$


A.13 The $\mathbf{Z}_{6}$ orbifold with $E_{8} \rightarrow E_{6} \times S U(2) \times U(1)$

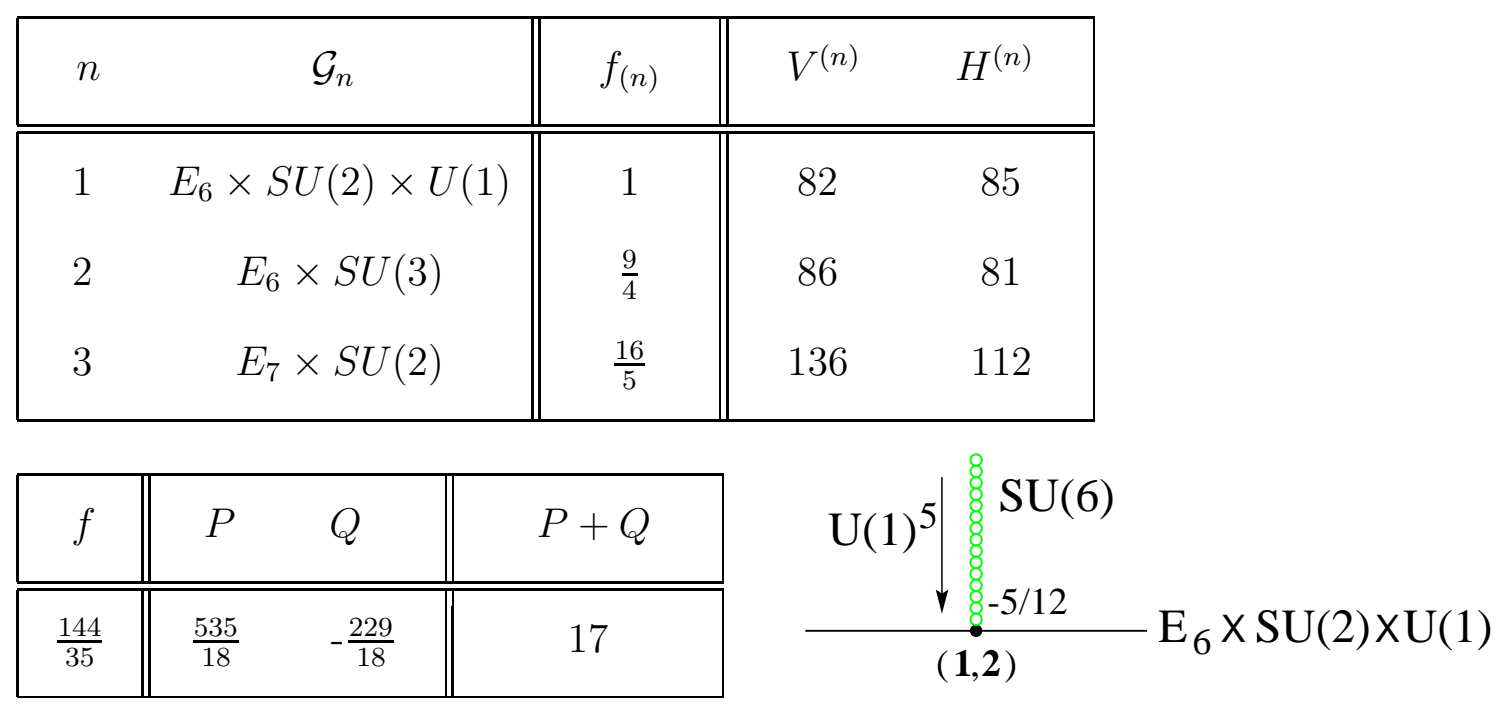

\begin{tabular}{|c||cc||ccc||ccccc||c|}
\hline$I$ & $\mathcal{G}_{I}$ & $\mathcal{R}$ & $\mathcal{I}_{4}$ & $\mathcal{I}_{2,2}$ & $\mathcal{I}_{2}$ & $n_{0}$ & $n_{1}$ & $n_{2}$ & $n_{3}$ & $\tilde{n}$ & $\mathrm{Z}$ \\
\hline \hline 1 & $E_{6}$ & $\mathbf{7 8}^{*}$ & 0 & $\frac{1}{2}$ & 4 & 1 & 1 & 1 & 1 & 0 & $\frac{35}{144}$ \\
& & $\mathbf{2 7}$ & 0 & $\frac{1}{12}$ & 1 & 6 & -3 & -3 & -2 & 0 & $-\frac{25}{24}$ \\
\hline 2 & $S U(2)$ & $\mathbf{3}^{*}$ & 0 & 8 & 4 & 1 & 1 & 1 & 1 & 0 & $\frac{35}{144}$ \\
& & $\mathbf{2}$ & 0 & $\frac{1}{2}$ & 1 & 56 & -29 & -25 & -56 & -1 & $-\frac{7}{18}$ \\
\hline
\end{tabular}

\begin{tabular}{|c||cccc||c|}
\hline$I$ & $X_{I}$ & $Z_{I}^{(4)}$ & $Z_{I}^{(2)}$ & $Z_{I}^{(2,2)}$ & $\rho_{I}$ \\
\hline \hline 1 & $\frac{1}{3}$ & 0 & $-\frac{5}{72}$ & $\frac{5}{144}$ & 0 \\
2 & 2 & 0 & $-\frac{5}{12}$ & $\frac{5}{4}$ & 0 \\
\hline
\end{tabular}

\begin{tabular}{|c||c|}
\hline$g$ & $\rho$ \\
\hline \hline$-\frac{5}{12}$ & 0 \\
\hline$n_{H}-n_{V}=\frac{9}{2}$
\end{tabular}


A.14 The $\mathbf{Z}_{6}$ orbifold with $E_{8} \rightarrow S U(9)$

\begin{tabular}{|cc||c||cc|}
\hline$n$ & $\mathcal{G}_{n}$ & $f_{(n)}$ & $V^{(n)}$ & $H^{(n)}$ \\
\hline \hline 1 & $S U(9)$ & 1 & 80 & 0 \\
2 & $S U(9)$ & $\frac{9}{4}$ & 80 & 84 \\
3 & $E_{8}$ & $\frac{16}{5}$ & 248 & 0 \\
\hline
\end{tabular}

\begin{tabular}{|c||cc||c|}
\hline$f$ & $P$ & $Q$ & $P+Q$ \\
\hline \hline$\frac{144}{35}$ & $\frac{535}{18}$ & $\frac{77}{18}$ & 34 \\
\hline
\end{tabular}

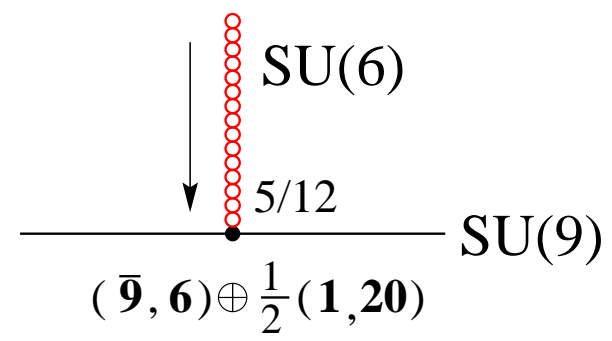

\begin{tabular}{|c||cc||ccc||ccccc||c|}
\hline$I$ & $\mathcal{G}_{I}$ & $\mathcal{R}$ & $\mathcal{I}_{4}$ & $\mathcal{I}_{2,2}$ & $\mathcal{I}_{2}$ & $n_{0}$ & $n_{1}$ & $n_{2}$ & $n_{3}$ & $\tilde{n}$ & $\mathrm{Z}$ \\
\hline \hline 1 & $S U(9)$ & $\mathbf{8 4}$ & -9 & 15 & 21 & 2 & 0 & -1 & 2 & 0 & $-\frac{13}{72}$ \\
& $\mathbf{8 0 ^ { * }}$ & 18 & 6 & 18 & 1 & 1 & 1 & 1 & 0 & $\frac{35}{144}$ \\
& $\mathbf{9}$ & 1 & 0 & 1 & 0 & 0 & 0 & 0 & -6 & 0 \\
\hline 1 & $S U(6)$ & $\mathbf{3 5}$ & 12 & 6 & 12 & 0 & 0 & 0 & 0 & $\frac{1}{2}$ & 0 \\
& $\mathbf{2 0}$ & -6 & 6 & 6 & 0 & 0 & 0 & 0 & $-\frac{1}{2}$ & 0 \\
& $\mathbf{6}$ & 1 & 0 & 1 & 0 & 0 & 0 & 0 & -9 & 0 \\
\hline
\end{tabular}

\begin{tabular}{|c||cccc||c|}
\hline$I$ & $X_{I}$ & $Z_{I}^{(4)}$ & $Z_{I}^{(2)}$ & $Z_{I}^{(2,2)}$ & $\rho_{I}$ \\
\hline \hline 1 & 2 & 0 & $-\frac{65}{12}$ & $-\frac{5}{4}$ & 0 \\
2 & 0 & 0 & -6 & 0 & 1 \\
\hline
\end{tabular}

\begin{tabular}{|c||c|}
\hline$g$ & $\rho$ \\
\hline \hline$\frac{5}{12}$ & 1 \\
\hline$n_{H}-n_{V}=\frac{93}{2}$
\end{tabular}


A.15 The $\mathbf{Z}_{6}$ orbifold with $E_{8} \rightarrow S U(6) \times S U(3) \times S U(2)$

\begin{tabular}{|c|c|c|c|c|c|c|c|c|c|c|c|}
\hline$n$ & \multicolumn{3}{|c|}{$\mathcal{G}_{n}$} & \multicolumn{2}{|r|}{$f_{(n)}$} & $V^{(n)}$ & \multicolumn{2}{|c|}{$H^{(n)}$} & & & \\
\hline 1 & \multicolumn{4}{|c|}{$S U(6) \times S U(3) \times S U(2)$} & 1 & 46 & \multicolumn{2}{|c|}{36} & & & \\
\hline 2 & \multicolumn{3}{|c|}{$E_{6} \times S U(3)$} & \multicolumn{2}{|r|}{$\frac{9}{4}$} & 86 & \multicolumn{2}{|c|}{81} & & & \\
\hline 3 & \multicolumn{3}{|c|}{$E_{7} \times S U(2)$} & \multicolumn{2}{|r|}{$\frac{16}{5}$} & 136 & \multicolumn{2}{|c|}{112} & & & \\
\hline$f$ & $P$ & $Q$ & \multicolumn{3}{|c|}{$P+Q$} & & \multicolumn{2}{|c|}{$\mathrm{SU}(6)$} & & & \\
\hline$\frac{144}{35}$ & $\frac{535}{18}$ & $\frac{5}{8}$ & \multicolumn{2}{|c|}{30} & & & \multicolumn{2}{|c|}{$-5 / 12$} & $\mathrm{U}(6$ & \multicolumn{2}{|c|}{$\begin{array}{l}\times \mathrm{SU}(3) \\
\times \mathrm{SU}(2)\end{array}$} \\
\hline$I$ & $\mathcal{G}_{I}$ & $\mathcal{R}$ & $\mathcal{I}_{4}$ & $\mathcal{I}_{2}$ & $\mathcal{I}$ & $n_{0}$ & $n_{1}$ & $n_{2}$ & $n_{3}$ & $\tilde{n}$ & $\mathrm{z}$ \\
\hline \multirow[t]{4}{*}{1} & $S U(6)$ & $35^{*}$ & 12 & 6 & 1 & 1 & 1 & 1 & 1 & $-\frac{1}{2}$ & $\frac{35}{144}$ \\
\hline & & 20 & -6 & 6 & ( & 2 & 0 & 2 & -2 & 0 & $-\frac{19}{72}$ \\
\hline & & 15 & -2 & 3 & 2 & 6 & 0 & -3 & 6 & 0 & $-\frac{13}{24}$ \\
\hline & & 6 & 1 & 0 & & 12 & -6 & -6 & -12 & 0 & $\frac{5}{72}$ \\
\hline \multirow[t]{2}{*}{2} & $S U(3)$ & $8^{*}$ & 0 & 9 & ( & 1 & 1 & 1 & 1 & 0 & $\frac{35}{144}$ \\
\hline & & 3 & 0 & $\frac{1}{2}$ & & 54 & -12 & -27 & 6 & 0 & $-\frac{15}{8}$ \\
\hline \multirow[t]{2}{*}{2} & $S U(2)$ & $3^{*}$ & 0 & 8 & 2 & 1 & 1 & 1 & 1 & 0 & $\frac{35}{144}$ \\
\hline & & 2 & 0 & $\frac{1}{2}$ & & 56 & -18 & 2 & -56 & 0 & $-\frac{25}{18}$ \\
\hline
\end{tabular}

\begin{tabular}{|c||cccc||c|}
\hline$I$ & $X_{I}$ & $Z_{I}^{(4)}$ & $Z_{I}^{(2)}$ & $Z_{I}^{(2,2)}$ & $\rho_{I}$ \\
\hline \hline 1 & 2 & 0 & $-\frac{77}{12}$ & $-\frac{19}{4}$ & 1 \\
2 & 2 & 0 & $-\frac{5}{12}$ & $\frac{5}{4}$ & 0 \\
3 & 2 & 0 & $-\frac{5}{12}$ & $\frac{5}{4}$ & 0 \\
\hline
\end{tabular}

\begin{tabular}{|c||c|}
\hline$g$ & $\rho$ \\
\hline \hline$-\frac{5}{12}$ & 1 \\
\hline
\end{tabular}




\section{References}

[1] P.Hořava and E.Witten, Heterotic and Type I String Dynamics from Eleven Dimensions, Nucl.Phys. B475 (1996) 94-114, hep-th/9510209.

[2] P.Hořava and E.Witten, Eleven-Dimensional Supergravity on a Manifold with Boundary, Nucl.Phys. B460 (1996) 506-524, hep-th/9603142.

[3] K.Dasgupta and S.Mukhi, Orbifolds of M-theory, Nucl.Phys. B465 (1996) 399-412, hep-th/9512196.

[4] E.Witten, Five-branes and M-Theory on an Orbifold, Nucl.Phys. B463 (1996) 383397, hep-th/9512219.

[5] L.Dixon, J.A.Harvey, C.Vafa and E.Witten, Strings on Orbifolds, Nucl. Phys. B261 (1985) 678, Strings on Orbifolds 2, Nucl. Phys. B274 (1986) 285.

[6] L.E.Ibañez, H.P.Nilles and F.Quevedo, Orbifolds and Wilson Lines, Phys. Lett. B187 (1987) 25; L.E.Ibañez, J.Mas, H.P.Nilles and F.Quevedo, Heterotic Strings in Symmetric and Asymmetric Orbifold Backgrounds, Nucl.Phys. B301 (1988) 157-196.

[7] M.Faux, D.Lüst and B.A.Ovrut, Intersecting Orbifold Planes and Local Anomaly Cancellation in M-theory, Nucl.Phys. B554 (1999) 437-483, hep-th/9903028

[8] M.Faux, D.Lüst and B.A.Ovrut, Local Anomaly Cancellation, M-theory Orbifolds and Phase-transitions, hep-th/0005251

[9] D.Lüst, String Vacua with N=2 Supersymmetry in Four Dimensions, Nucl. Phys. B (Proc. Suppl) 62 (1998) 375, hep-th/9803072.

[10] Adel Bilal, Jean-Pierre Derendinger, Roger Sauser, M-Theory on $S^{1} / Z_{2}:$ New Facts from a Careful Analysis, hep-th/9912150.

[11] V. Kaplunovsky, J. Sonnenschein, S. Theisen, S. Yankielowicz, On the Duality between Perturbative Heterotic Orbifolds and $M$-Theory on $T^{4} / Z_{N}$, hep-th/9912144.

[12] G.Aldazabal, A.Font, L.E.Ibáñez, A.M.Uranga and G.Violero, Non-Perturbative Heterotic $D=6, N=1$ Orbifold Vacua, Nucl.Phys. B519 (1998) 239-281, hep-th/9706158

[13] A. Lukas, B. A. Ovrut, K. S. Stelle and D. Waldram, The Universe as a Domain Wall, Phys.Rev. D59 (1999) 086001; Heterotic M-theory in Five Dimensions, Nucl.Phys. 
B552 (1999) 246-290; A. Lukas, B. A. Ovrut and D. Waldram, Non-Standard Embedding and Five-Branes in Heterotic M-Theory, Phys.Rev. D59 (1999) 106005; R. Donagi, A. Lukas, B. A. Ovrut and D. Waldram, Non-Perturbative Vacua and Particle Physics in M-Theory, JHEP 9905 (1999) 018; Holomorphic Vector Bundles and Non-Perturbative Vacua in M-Theory, JHEP 9906 (1999) 034; A. Lukas, B. A. Ovrut and D. Waldram, Five-Branes and Supersymmetry Breaking in M-Theory, JHEP 9904 (1999) 009; R. Donagi, B. A. Ovrut and D. Waldram, Moduli Spaces of Fivebranes on Elliptic Calabi-Yau Threefolds, JHEP 9911 (1999) 030; R. Donagi, B. A. Ovrut, T. Pantev and D. Waldram, Standard Models from Heterotic M-theory, hepth/9912208; R.Donagi, B.Ovrut, T.Pantev and D.Waldram, Standard Model Bundles on Non-Simply Connected Calbi-Yau Threefolds, hep-th/0008008; R.Donagi, B.Ovrut, T.Pantev and D.Waldram, Standard Model Bundles, math.AG/0008010; R.Donagi, B.Ovrut and D.Waldram, Spectral Involutions on Rational Elliptic Surfaces, math.AG/0008011.

[14] B. A. Ovrut, T. Pantev and J. Park, Small Instanton Transitions in Heterotic MTheory, hep-th/0001133.

[15] R.Slansky, it Group Theory for Unified Model Building, Phys.Rep. 79 (1981) 1-128.

[16] T. van Ritbergen, A.N. Schellekens, J.A.M. Vermaseren, Group theory factors for Feynman diagrams, Int.J.Mod.Phys. A14 (1999) 41-96, hep-ph/9802376. 